\title{
COLLISION CROSS SECTIONS OF GAS PHASE DNA IONS
}

by

\author{
ANNIE MORADIAN \\ B.Sc., Sharif University of Technology, 1994

\begin{abstract}
A THESIS SUBMITTED IN PARTIAL FULFILMENT OF THE REQUIREMENT FOR THE DEGREE OF

MASTER OF SCIENCE
\end{abstract} \\ in \\ THE FACULTY OF GRADUATE STUDIES \\ Department of Chemistry
}

We accept this thesis as confirming

to the required standard

THE UNIVERSITY OF BRITISH COLUMBIA

July 2002

(C) Annie Moradian, 2002 
in presenting this thesis in partial fulfilment of the requirements for an advanced degree at the University of British Columbia, I agree that the Library shall make it freely available for reference and study. I further agree that permission for extensive copying of this thesis for scholarly purposes may be granted by the head of my department or by his or her representatives. It is understood that copying or publication of this thesis for financial gain shall not be allowed without my written permission.

Department of Chemistay

The University of British Columbia Vancouver, Canada

Date 225 ely $/ 2002$ 


\section{Abstract}

The aim of this work is to study the collision cross sections of single stranded DNA ions in an electrospray ionization (ESI) mass spectrometer (MS). In part this work was motivated by a fall-off in signal intensities in ESI-MS with increasing molecular weight of the DNA. The first

part of this thesis discusses the theories and experimental methods of measuring and calculating collision cross sections of bio-molecules. The collision cross section is a measure of ion "size" which can provide direct insight to structural characterization of bio-molecules. The second part of this thesis describes experiments with 28-, 40- and 55-mer oligodeoxynucleotide ions and protein ions of nearly the same molecular weight - ubiquitin, cytochrome $c$ and apomyoglobin. The energy losses of ions from collisions with argon were measured and interpreted with a diffuse scattering model. The last part of this thesis presents the comparison of cross sections of oligodeoxynucleotides with cross sections of the proteins. The oligodeoxynucleotides produce negative charge states in ESI similar to he positive charge states produced by the proteins denatured in solution. Cross sections for oligodeoxynucleotide ions increase with charge state similar to those of protein ions. For a given molecular weight and charge state, the cross sections of the oligodeoxynucleotide ions are about $22 \%$ lower than those of the proteins. In this study, ESI mass spectra of 28-, 40- and 55-mer oligodexoynucleotides show similar sensitivities and a fall-off in signal intensity with increasing mass has not been observed. 


\section{Table of Contents}

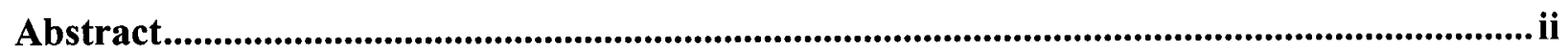

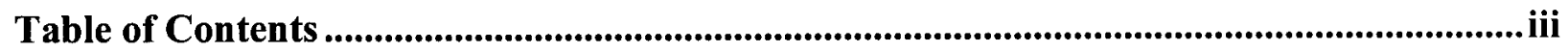

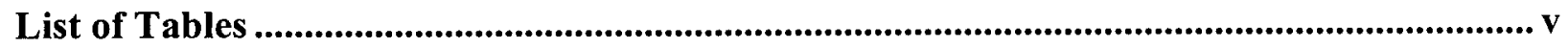

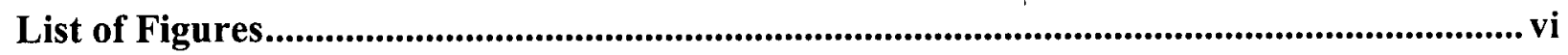

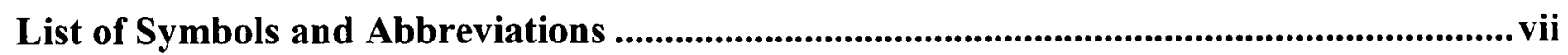

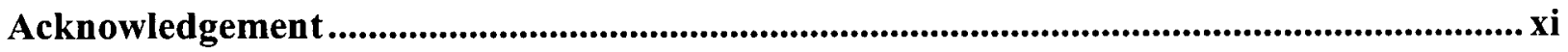

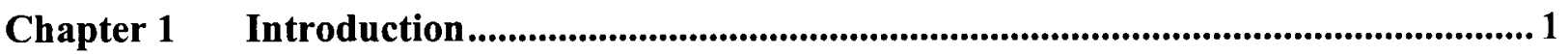

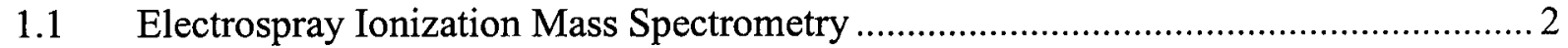

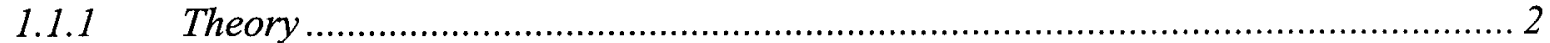

1.1.2 The Properties of Electrosprayed Ions ............................................................. 5

1.1:3 ESI-MS Interface ............................................................................. 5

1.2 Studies of Gas-Phase Bio-Molecules by ESI-MS ......................................... 6

1.2.1 ESI - MS of Proteins........................................................................ 6

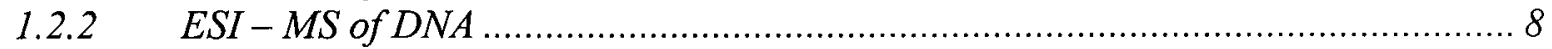

1.3 Practical Methods for Studying Gas-Phase Bio-Molecules by Mass Spectrometry.... 11

1.3.1 Proton Transfer Reaction ...................................................................... 11

1.3.2 Hydrogen-Deuterium (H/D) Exchange ..................................................... 13

1.3.3 Collision Cross Section Measurements ...................................................... 15

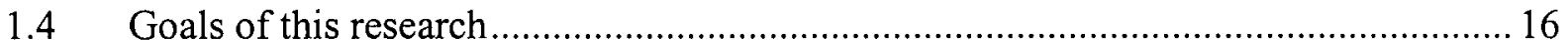

Chapter 2 Collision Cross Sections of Gas Phase Bio-Molecules .................................... 18

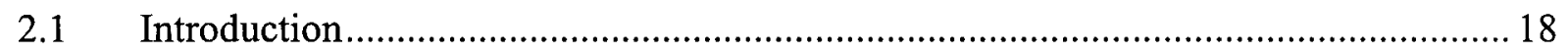

2.2 Experimental Methods for Measuring Collision Cross Sections............................ 19

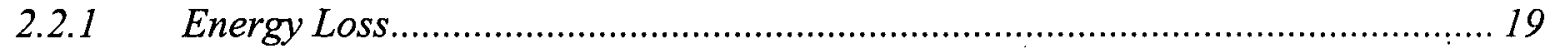

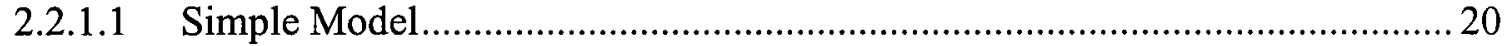

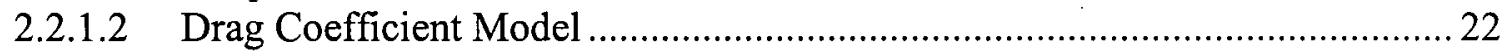

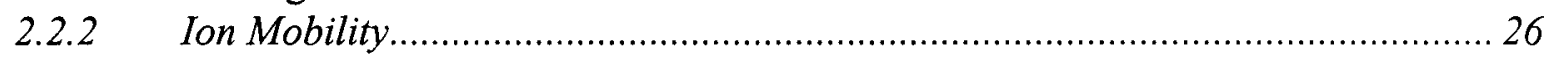

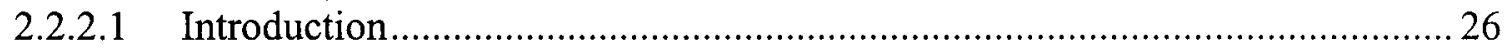

2.2.2.2 Hard Sphere Model .................................................................................. 28

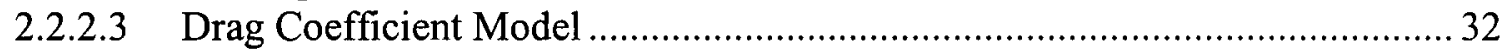

2.2.3 Energetic Surface Imprinting.......................................................... 33

Chapter 3 Instrumentation, Experimental Procedures and Sample Preparation ......... 36

3.1 Electrospray Ionization Triple Quadrupole Mass Spectrometer................................ 36

3.1.1 Operation Principles of Quadrupole Mass Filters ......................................... 36

3.1.2 Electrospray Ionization Triple Quadrupole Mass Spectrometer........................ 39 
3.1.3 Reduced Liquid Flow Rate Electrospray Source .......................................... 41

3.1.4 Ion Detection.................................................................................... 41

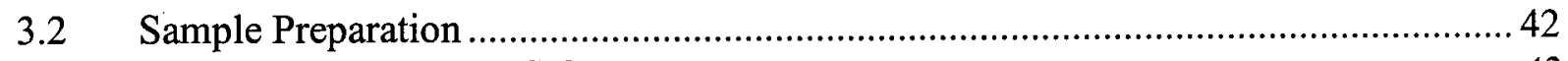

3.2.1 DNA and Protein Solutions................................................................ 42

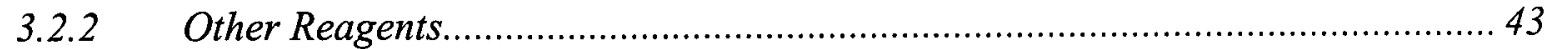

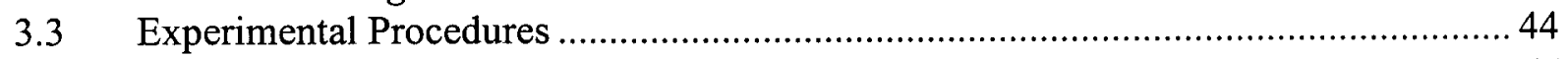

3.3.1 Measurements of Ion Energy Loss ........................................................ 44

3.3.2 Data Analysis of Energy Loss Experiments ............................................ 45

Chapter 4 Collision Cross Sections of DNA and Protein Ions .........................................48

4.1 Mass spectra and sensitivities of 28, 40 and 55-mer Oligodeoxynucleotides ............48

4.1.1 Mass spectra of 28-, 40 and 55-mer oligodeoxynucleotides............................. 48

4.1.2 Sensitivity Comparisons of the 28-, 40- and 55-mer Oligodeoxynucleotides...... 51

4.2 Mass Spectra of Ubiquitin, Cytochrome $c$ and Myoglobin ...................................53

4.3 DNA and Protein Ion Cross Sections....................................................... 56

Chapter 5 Future Work....................................................................................................66

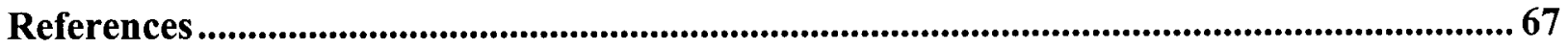




\section{List of Tables}

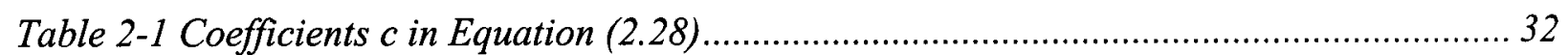

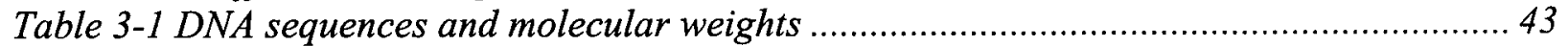

Table 3-2 Voltages used in Energy Loss Experiments........................................................ 45

Table 4-1 Drag Coefficients of 28-, 40- and 55-mer Oligodeoxynucleotide Ions ..................... 57

Table 4-2 Drag Coefficients of Ubiquitin, Cytochrome $c$ and Myoglobin Ions........................ 58 


\section{List of Figures}

Figure 1-1 Schematic view of an electrospray ionization source 2 Figure 1-2 Mass spectra of cytochrome c 9:1 ( $\left.\mathrm{H}_{2} \mathrm{O}: \mathrm{MeOH}\right)$. Peaks from m/z 1237 through 1766 are multiply protonated $(+7$ to +10$)$ ions

Figure 1-3 Structure of a part of a DNA chain__ 9

Figure 2-1 General schematic of ion mobility measurement instrument 29

Figure 2-2 Schematic diagram of MUMMA. ESI=electrospray ionization; $Q M S=$ quadrupole mass spectrometer; $H . V .=$ high voltage; $M C P=$ multichannelplate detector; $T=$ target. $\ldots 34$

Figure 3-1 Conventional circular quadrupole rods in a quadrupole mass filter. _____ 37

Figure 3-2 The first stability region of a quadrupole field. $m_{1}, m_{2}$ and $m_{3}$ represents ions with different masses on an operating line.

Figure 3-3 Schematic of the ESI-triple quadrupole mass spectrometer system: S, electrospray ionization source; $N_{2}$, nitrogen curtain gas; $C$, curtain plate; $O$, sampling orifice; $S K$, skimmer; $Q 0$, rf only quadrupole; $P F$, prefilter; $Q 1$, mass-analyzing quadrupole; $Q 2$, collision cell; $Q 3$, mass-analyzing quadrupole; $D$, detector. 40

Figure 3-4 Schematic diagram of reduced liquid flow rate ESI source. 1-tapered tip; 2 stainless steel junction; 3-fused silica capillary; 4- epoxy glue. 41

Figure 3-5 Ion detection circuit for negative ions.

Figure 3-6 The stopping curves of 28-mer (-5) ions at different cell pressures of Ar. The curves from right to left correspond to cell pressures of $0,0.275,0.581,0.853$ and 1.141 militorr of Ar, respectively. " 0 " means no added collision gas.

Figure 3-7 A plot of E/EO vs. -(CD l m2 n)/ml for 28-mer (-5) ion.

Figure 4-1 Mass spectrum of the 28-mer oligodeoxynucleotide. 49

Figure 4-2 Mass spectrum of the 40-mer oligodeoxynucleotide. 50

Figure 4-3 Mass spectrum of the 55-mer oligodeoxynucleotide. 51

Figure 4-4 Mass spectrum of ubiqutin.

Figure 4-5 Mass spectrum of cytochrome c.

Figure 4-6 Mass spectrum of myoglobin.

Figure 4-7 Collision cross section vs. charge state of ions of ubiquitin and 28-mer.

Figure 4-8 Collision cross section vs. charge state of ions of cytochrome $c$ and 40-mer.

Figure 4-9 Collision cross section vs. charge state of ions of apomyoglobin and 55-mer.

Figure 4-10 Cross section per nucleotide vs. charge per nucleotide for ions of the 28-, 40- and

55-mer. 60

Figure 4-11 Cross section / (number of nucleotides) ${ }^{2 / 3}$ vs. charge / (number of nucleotides) ${ }^{2 / 3}$ for ions of the 28-, 40-and 55-mer. 


\section{List of Symbols and Abbreviations}

\begin{tabular}{|c|l|}
\hline $\begin{array}{c}\text { Symbol or } \\
\text { Abbreviation }\end{array}$ & Definition \\
\hline ESI & Electrospray ionization \\
rf & Electron impact ionization \\
dc & Radiofrequency \\
MALDI & Direct current \\
CRM & Matrix assisted laser desorption ionization \\
IEM & Ion evaporation model \\
r & Droplet radius \\
LC & Liquid chromatography \\
CE & Capillary electrophoresis \\
MS & Mass spectrometry \\
TOF & Time of flight \\
TEA & Triethylamine \\
PCR & Polymerase chain reaction \\
$\Delta H$ & Enthalpy change \\
$\Delta G$ & Free energy change \\
GB & Gas phase basicity \\
PA & Proton affinity \\
\hline & Nuclear magnetic resonance \\
\hline
\end{tabular}




\begin{tabular}{|c|c|}
\hline $\mathrm{H} / \mathrm{D}$ & Hydrogen / Deuterium \\
\hline FTICR & Fourier transform ion cyclotron resonance \\
\hline $\mathrm{MS} / \mathrm{MS}$ & Mass spectrometry/mass spectrometry \\
\hline $\mathrm{CAD}$ & Collisionally activated dissociation \\
\hline Q0 & Quadrupole ion guide \\
\hline Q1 & First quadrupole \\
\hline Q2 & Second quadrupole \\
\hline Q3 & Third quadrupole \\
\hline $\mathrm{E}_{\mathrm{lab}}$ & Laboratory energy before collision \\
\hline$E^{\prime}{ }_{\text {lab }}$ & Laboratory energy after collision \\
\hline $\mathrm{m}_{1}$ & Ion mass \\
\hline $\mathrm{m}_{2}$ & Neutral collision partner mass \\
\hline $\mathrm{E}_{\text {int }}$ & Internal energy \\
\hline$\theta_{\mathrm{cm}}$ & Scattering angle in center-of-mass coordinates \\
\hline $\mathrm{E}_{1}$ & Laboratory energy after one collision \\
\hline $\mathrm{E}_{0}$ & Laboratory energy before any collisions \\
\hline$\alpha$ & $\mathrm{E}_{1} / \mathrm{E}_{0}$ \\
\hline $\mathrm{N}$ & Number of collisions \\
\hline 1 & Length of collision cell \\
\hline $\mathrm{n}$ & Number density \\
\hline$\lambda$ & Mean free path \\
\hline$\sigma$ & Collision cross section \\
\hline S & Target thickness \\
\hline
\end{tabular}




\begin{tabular}{|c|c|}
\hline$\beta$ & $1 / \alpha$ \\
\hline $\mathrm{F}_{\mathrm{D}}$ & Drag force \\
\hline$v$ & Ion speed \\
\hline $\mathrm{C}_{\mathrm{Dd}}$ & Drag coefficient for diffuse scattering \\
\hline $\mathrm{C}_{\mathrm{Ds}}$ & Drag coefficient for specular scattering \\
\hline s & Ratio of an object's speed to the thermal speed of a gas \\
\hline $\mathrm{T}$ & Gas temperature \\
\hline $\mathrm{k}_{\mathrm{B}}$ & Boltzman's constant \\
\hline $\mathrm{K}_{\mathrm{n}}$ & Knudsen's number \\
\hline D & Diameter \\
\hline $\mathrm{R}$ & Reynolds number \\
\hline$\rho$ & Gas density \\
\hline$\eta$ & Viscosity \\
\hline$v_{\mathrm{d}}$ & Drift velocity \\
\hline $\mathrm{K}$ & Mobility \\
\hline$t_{d}$ & Drift time \\
\hline $\mathrm{E}$ & Electric field strength \\
\hline $\mathrm{K}_{0}$ & Reduced mobility \\
\hline $\mathrm{P}$ & Gas pressure \\
\hline $\mathrm{n}_{0}$ & Number density in standard conditions \\
\hline $\mathrm{z}$ & Number of charges \\
\hline$\Omega^{1,1}$ avg & Orientationally averaged collision integral \\
\hline
\end{tabular}




\begin{tabular}{|c|c|}
\hline $\mathrm{b}_{\min }$ & Minimum impact parameter \\
\hline$\theta, \phi, \gamma$ & Three angles that define collision geometry \\
\hline$\chi(\theta, \phi, \gamma, \mathrm{b})$ & Scattering angle \\
\hline EHSS & Exact hard sphere scattering model \\
\hline A & Projection area \\
\hline e & Electron charge \\
\hline $\mathrm{q}$ & $\mathrm{ze}$ \\
\hline MUMMA & Multiply charged macromolecular accelerator \\
\hline $\mathrm{L}^{\prime}$ & Projected length of the protein \\
\hline $\mathrm{L}$ & Molecular length \\
\hline $\mathrm{U}$ & Direct current voltage \\
\hline$\omega$ & Angular frequency \\
\hline CEM & Channel electron multiplier \\
\hline HFIP & 1,1,1,3,3,3,-hexafluoro-2-propanol \\
\hline HPLC & High performance liquid chromatography \\
\hline BPTI & Bovine pancreatic trypsin inhibitor \\
\hline $\mathrm{N}_{\mathrm{i}}$ & Number of charges of a peak in a spectrum \\
\hline$I_{i}$ & Relative intensity of a peak in a spectrum \\
\hline
\end{tabular}




\section{Acknowledgement}

This thesis would not exist without many individuals.

I would like to thank my supervisor Don Douglas for guiding and supporting me from the beginning through the end of this research. Being in Don's group was a privilege and joy for me. I am indebted to Dunmin for being such a great help, no matter when I needed it, for all our discussions and his insightful suggestions. I am grateful to Brad for teaching me how to use the triple quadrupole and making ESI tips for me, I appreciate his kindness. I would like to extend my gratitude to Josh, Ravi, Yu-Luan, ChuanFan, Wei, Samir, Aaron, Kevin and Milica, you made my stay in UBC a memorable one. I gratefully acknowledge NSERC and Sciex for financial support of this research and Lloyd Smith, Michael Westphall and Mark Scalf, Chemistry Department University of Wisconsin, for their collaboration on this project.

I appreciate my father-in-law and mother-in-law for all their kindness, help and support. I would like to thank my mother for all her sacrifices, for teaching me the meaning of hard work and endurance and for showing me how to appreciate life through her example, my brother for always being there for me, my lovely daughter Beaina, for giving my life a new meaning and my husband Ara for his love, faith, encouragement and most of all his friendship that is my inspiration. This thesis is dedicated to them. 


\section{Chapter 1 Introduction}

The first mass spectrometer was built in the early 1900's by J.J. Thomson [1]. He used fixed magnetic and electric fields to separate ions of different mass and energy and was able to observe that the noble gas $\mathrm{Ne}$ was composed of two different isotopes of masses $20 \mathrm{Da}$ and 22 $\mathrm{Da}$, contributing to the final proof of the atomic theory of matter. The first practical mass spectrometers were made available in the early 1940's and were principally used for low mass organic analysis. These instruments used electron impact ionization (EI) to produce analyte ions. The basic principles of the quadrupole mass filter were published in the early 1950's by Paul and co-workers [2]. Mass separation in a quadrupole mass filter is based on achieving a stable trajectory for ions of specific $\mathrm{m} / \mathrm{z}$ values in a quadrupolar electric field with radiofrequency (rf) and direct-current (dc) components. Quadrupole mass analyzers have been used in conjunction with EI sources since their invention.

Over the last two decades several ionization techniques such as electrospray ionization (ESI) [3] and matrix assisted laser desorption ionization (MALDI) [4] have been introduced and have turned mass spectrometry into a powerful method for characterization of biological molecules. Today, substances in the attomole/ $\mu$ l range (about $10^{-12} \mathrm{M}$ ) can be detected, and molecular masses of biomolecules over 100,000 Da can be measured. 


\subsection{Electrospray Ionization Mass Spectrometry}

\subsubsection{Theory}

Electrospray Ionization (ESI) is a technique that allows the transfer of ions from solution to the gas phase. The dispersion of a liquid into electrically charged droplets and ions happens in three major steps: Generation and charging of the droplets, droplet shrinkage and evaporation, and finally gaseous ion formation. Figure 1-1.

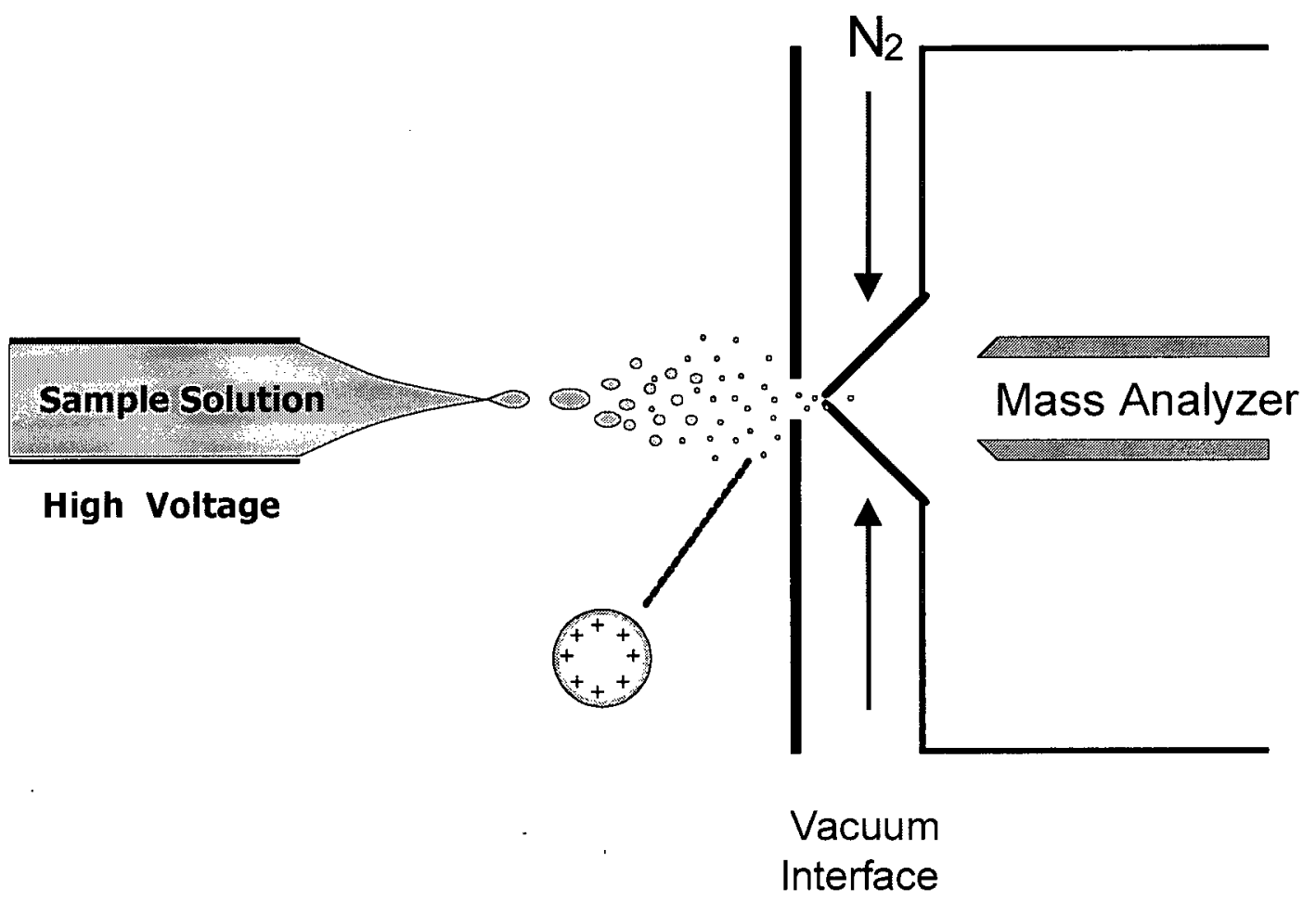

Figure 1-1 Schematic view of an electrospray ionization source

An applied very high electric field at an electrospray metal capillary tip leads to a partial separation of positive and negative electrolyte ions in the solution. In the positive ion mode, positive ions accumulate at the surface of the liquid at the capillary tip. Consequently Coulomb 
elongates into a filament or jet that breaks up into individual charged droplets. As the droplets migrate toward a counter-electrode, solvent evaporates and the droplets become smaller, while the charge they carry remains constant. They approach the Rayleigh limit where they become unstable and undergo "Coulomb fission", a process that leads to the production of smaller offspring droplets. This sequence of events repeats, resulting in very small highly charged droplets.

Currently there are two theories to explain the formation of gas phase ions, a Charged Residue Model (CRM) and an Ion Evaporation Model (IEM). The first initially assumed by Dole [5] and later modified by Röllegan [6], suggests that if the original concentration of analyte is sufficiently low, repeated Coulomb fission produces droplets that contain only a single analyte molecule. This analyte molecule will retain the residual droplet charge as the last solvent molecules evaporate, thus forming a gas-phase ion.

The IEM mechanism proposed by Iribarne and Thomson [7] assumes that when solvent evaporation and Coulomb droplet fission have reduced the size of the charged droplets to a certain radius $\mathrm{r}(\mathrm{r} \sim 10 \mathrm{~nm})$, the electric field on the surface of the charged droplets is sufficiently high that, direct emission of ions to the gas phase begins to occur.

It is assumed in both theories that droplets with radii $\mathrm{r}>10 \mathrm{~nm}$ release Coulombic stress by Rayleigh fission and for the droplets with radii $\mathrm{r}<1 \mathrm{~nm}$ neither the Rayleigh condition nor the ion evaporation theory applies [8].

The details of these mechanisms and their implications are a continued focus of study and debate, and, with the available evidence, it is not possible to state with certainty which theory fits better. Dole and co-workers [5] first introduced the idea of producing gas-phase ions from high molecular weight polymers by electrospray in 1968. Since conventional mass 
analyzers available at the time could not detect ions of such high mass, the experimental results obtained were not conclusive. Over a decade later Yamashita and Fenn [9] mass analyzed ions by interfacing an atmospheric pressure electrospray source to a quadrupole mass analyzer, and it took only a few years before the importance of the method was recognized $[10,11]$.

A conventional ESI 'source' is constructed from stainless-steel tubing, or a coaxial arrangement of fused silica and stainless-steel capillaries, incorporating a method for electrically biasing the liquid solution relative to a counter electrode. Modification to the simple ESI system has been done in order to improve the nebulization and desolvation processes with the assistance of a high-velocity gas flow. This variation is called 'ion spray' [12]. It was originally employed to make ESI compatible with flows from a conventional liquid chromatography (LC) column. A sheath flow of organic solvents coaxial to the sample solution, which was first used for the interfacing of capillary electrophoresis with ESI-MS [13], is another method used to improve ESI performance.

Low solution flow rate ESI sources like microspray and nanospray require a miniaturized version of the fused silica capillary with narrower inside and outside diameter (10$50-\mu \mathrm{m}$ ) to ensure flow rates as low as $25 \mathrm{~nL} / \mathrm{min}$ [14]. This has lowered the sample size required for ESI detection, pushing the limit to pmol and fmol quantities. ESI is used for many analytes including biomolecules, organic acids and bases, organometallics, metal complexes, natural products, and many others. 


\subsubsection{The Properties of Electrosprayed Ions}

Electrospray ionization (ESI) has the following features that make it one of the most widely used sources for ionization:

(i) The transfer of ions to the gas phase is not an energetic process; therefore ions with low internal energy are produced. These ions are less likely to be fragmented and even noncovalently bound complexes can be detected by the mass spectrometer.

(ii) Multiply charged ions are produced. This results in relatively low mass-to-charge $(\mathrm{m} / \mathrm{z})$ ratios for macromolecules.

(iii) The natural compatibility of ESI with solutions allows it to be coupled easily with several separation techniques, including LC and CE.

\subsubsection{ESI - MS Interface}

The requirement of an ESI - MS interface is to sample and transport ions from the highpressure region into the vacuum system of a mass spectrometer as efficiently as possible. At atmospheric pressure, ions drift under the influence of the electric field between the capillary tip and an interface plate. Some systems employ a capillary-inlet skimmer system as described by Fenn and co-workers [15]. Generally the instrument utilizes differential pumping to increase the ion sampling efficiency. To assist evaporation of the droplets and the break up of unwanted cluster ions, a drying gas (nitrogen) sometimes referred to as 'curtain' gas is arranged to flow between the interface plate and the orifice. A heated transfer-tube system described by Chait et al. [16] can also be used for desolvation and ion transport. In many interface designs, transport of ions from atmospheric pressure to vacuum results in a free jet expansion, and ions can then be sampled by a skimmer into the next vacuum stage. In this stage the pressure is lower, but still 
not low enough for effective focusing with ion lenses. Quadrupole, hexapole, and octapole ion guides are now widely used to transport ions into the mass analyzer section. Douglas and French [17] have observed that the efficiency of guiding ions is significantly increased if the pressure is

raised to $10^{-2}$ Torr. Ions are slowed by collisions in the rf-only quadrupole, move to the centre of the quadrupole, and enter the next stage with a limited energy spread of a few electron volts.

\subsection{Studies of Gas-Phase Bio-Molecules by ESI-MS}

Knowledge of a bio-molecule's structure is important for understanding and predicting its function. With the rapid development of molecular biology and its impact on all areas of life sciences, as well as with the development of the biotechnology industry, analytical methods are needed to determine the structure and purity of these bio-molecules and their constituents. ESIMS has proven to be one such method.

\subsubsection{ESI - MS of Proteins}

Determining the molecular weight of a protein is a rapid method to confirm the identity of a sample. As first reported by Fenn and co-workers [18] the molecular weight $\mathrm{M}$ of a macromolecule may be immediately determined from spectra such as, given the following assumptions:

1- The adjacent peaks of a series differ by only one charge.

2- Charging is due to proton attachment to the molecular ion. 


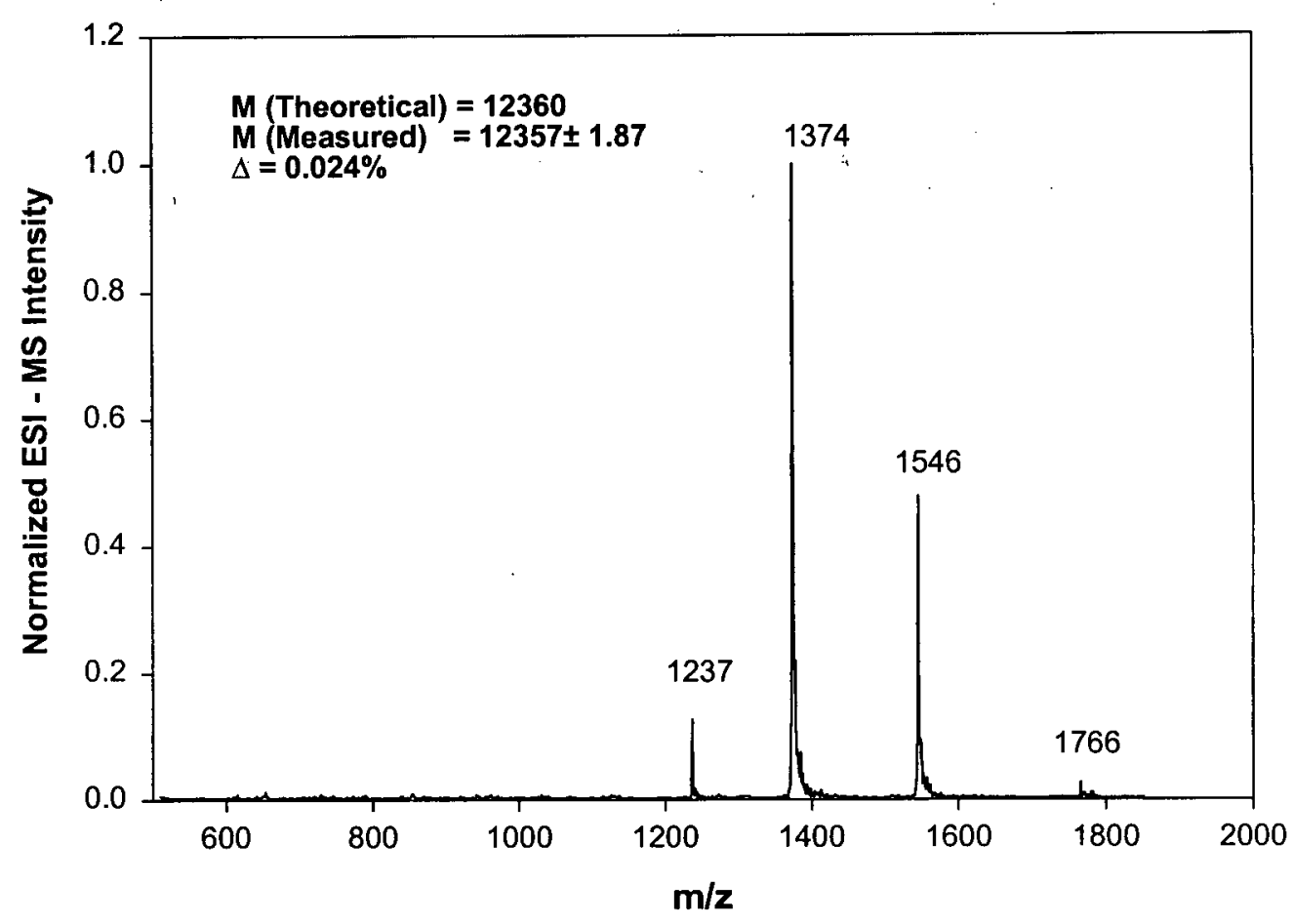

Figure 1-2. Mass spectra of cytochrome $c$ 9:1 $\left(\mathrm{H}_{2} \mathrm{O}: \mathrm{MeOH}\right)$. Peaks from m/z 1237 through 1766 are multiply protonated $(+7$ to +10$)$ ions

Protein folding is the process in which the linear information contained in the amino acid sequence of a polypeptide chain is translated into a defined three-dimensional structure. The three dimensional structures of folded proteins result from a combination of non-covalent interactions including, short-range repulsion, electrostatic forces, van der waals interactions and hydrogen bonding. In their native state, globular proteins have tightly folded, compact structures. The denatured state of a protein is a drastic change in this structure without alteration of the amino acid sequence, but invariably a loss of biological function. Proteins can be denatured by subjecting them to high temperatures, extremes of $\mathrm{pH}$, and solutions containing high concentrations of compounds such as urea, and organic solvents [19]. Chait et al [20] demonstrated that conformational changes in proteins could also be detected by ESI-MS, 
because the three-dimensional structure of a protein influences the charge distribution in its spectrum.

In solution, the net charge on the protein will depend on the number of accessible basic (Arg, Lys, His, $\mathrm{NH}_{2}$-terminus) and acidic sites (Asp, Glu, Tyr, $\mathrm{COOH}$ terminus) and the solution $\mathrm{pH}$. It has been suggested that protonation occurs at basic residues for positive ions and deprotonation at acidic residues for negative ions.

Generally an unfolded protein shows higher charge states in its ESI-MS spectrum than the same protein in the native state. This has been confirmed for various proteins and different denaturing agents such as heat [21], acid [22,23], solution composition $[24,25]$ and reduction of disulfide bonds [26]. The change in charge state has been attributed to changes in the steric accessibility of protonation sites, as well as the increasing surface area of the unfolded state. Time resolved mass spectrometry [27] has also been used to study the changes in protein conformations during unfolding reactions.

Charge state distributions found in ESI mass spectra depend on several factors, including the charge distribution in the solution prior to analysis, the efficiency for production of charged ions during the spray and ion desorption processes, and the ion transmission and detection efficiencies of the mass analyzer.

\subsubsection{ESI - MS of DNA}

DNA is a linear (or circular) copolymer of nucleotides. Nucleotides have three characteristic components: (1) a nitrogenous base, (2) a pentose, and (3) a phosphate. Four heterocyclic bases (nucleobases); the purines adenine (A) and guanine (G), and the pyrimidines cytosine (C), thymine $(\mathrm{T})$ are predominant in DNA structure [28]. The successive nucleotides 
are covalently linked through phosphate-group bridges. Specifically, the 5'-hydroxyl group of one nucleotide unit is joined to the $3^{\prime}$-hydroxyl group of the next nucleotide by a phosphodiester linkage as is shown in Figure 1-3.

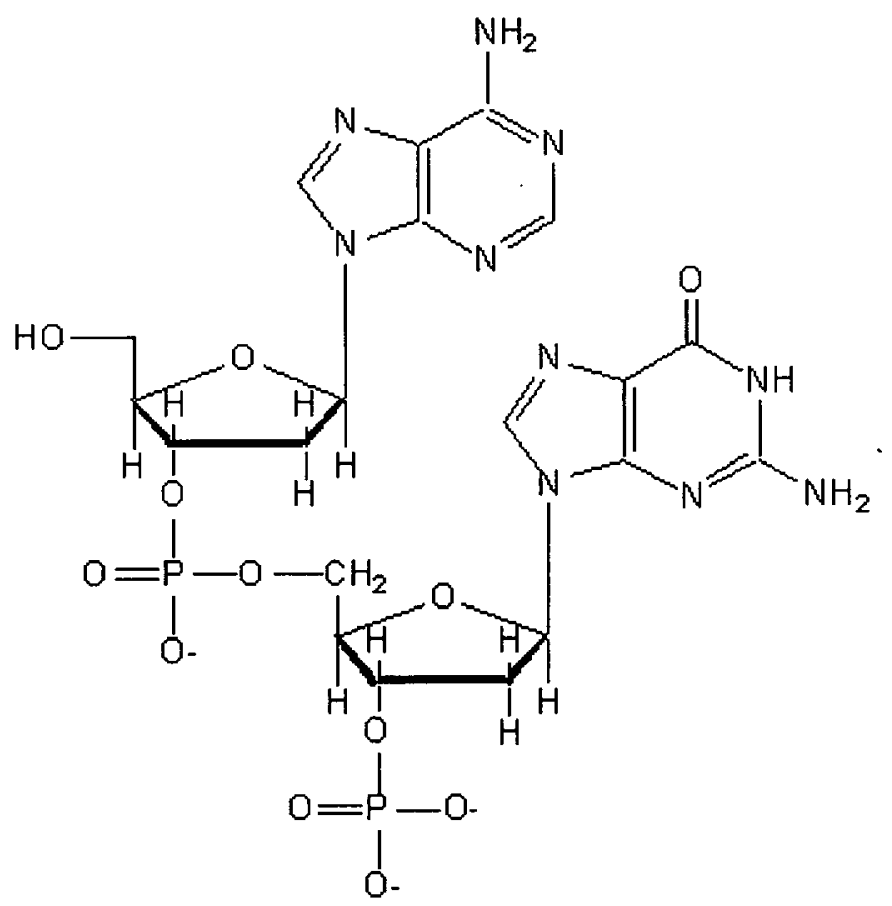

Figure 1-3 Structure of a part of a DNA chain

The advent of ESI-MS and MALDI-Time of Flight Mass Spectrometry (TOF-MS) enabled the analysis of higher molecular weight nucleic acids and their constituents. Covey et al. [10] first demonstrated the formation of negative ions of a 14-mer nucleotide and measured the molecular weight. This work was followed by Smith and co-workers [29] who electrosprayed oligonucleotides up to 72-mer.

Oligonucleotides possess an acidic proton at each phosphodiester bond along their backbone $\left(\mathrm{pK}_{\mathrm{a}}<1\right)$. In solution, solvent acts as a dielectric shield to reduce repulsive Coulombic interactions that results from the negative charge of -1 at every nucleotide residue. The process of transferring the analyte from solution into the gas-phase results in the loss of 
solvent and adduction of cations present in the solution. In ESI-MS The main difficulty arises from formation of oligonucleotide-sodium or potassium adducts which can make the spectra very complex.

Therefore, several procedures have been developed to remove or displace these adducts. One of the earlier adopted procedures was to remove adducts by ammonium ions, which appear to be less tightly bound to the nucleotides when ionized in the gas phase. Replacing sodium and potassium adducts with ammonium can be accomplished by repeated cold ethanol precipitation of oligonucleotides from concentrated ammonium acetate solutions [30]. Other methods include, precipitation from ammonium acetate in conjunction with the addition of organic additives and chelating agents [31] and addition of strong organic bases such as triethylamine (TEA) that effectively displace the cation [32].

Few studies have been performed on the charge state distributions of oligonucleotides in ESI-MS. Tong and co-workers [33] studied charge state distributions for hexadeoxynucleotides and concluded that there are clear differences in the maximum charge states of these nucleotides as a function of $\mathrm{pH}$. This indicates a correlation of charge state, with the basicity $\left(\mathrm{pK}_{\mathrm{a}}\right)$ of nucleotide bases. Griffey and co-workers [34] reported that the charge states of oligonucleotides in ESI are a function of the concentration of ammonium ions, if present in the sample. A reduction in average charge state is observed with increasing solution concentrations of ammonium acetate.

ESI-MS can be used without initial separation to determine the molecular weight of oligonucleotides of moderate size in simple mixtures but for the analysis of more complex mixtures from in vivo experiments or PCR products the use of a separation technique such as HPLC in conjunction with ESI-MS is necessary. Appfel et al [35] described a reversed phase- 
online HPLC-ESI-MS separation using a mobile phase containing $400 \mathrm{mM} \mathrm{1,1,1,3,3,3}$

hexafluoro-2-isopropanol adjusted to $\mathrm{pH} 7.0$ with TEA in a methanol gradient $(0-20 \%)$ which resulted in efficient HPLC separation and high sensitivity ESI-MS with minimum adduct formation. Using ESI-MS the molecular weights of oligonucleotides with lengths of over 100 bases can now be measured to an accuracy of $50 \mathrm{ppm}[36]$.

\subsection{Practical Methods for Studying Gas-Phase Bio-Molecules by Mass Spectrometry}

ESI-MS provides a unique opportunity to study intact protein molecules in the gas phase, without any solvent effects. Once bio-molecules are introduced into the gas phase, it is possible to probe them by chemical or physical methods in order to gain information about their structures. Several methods have been developed for this purpose. This section includes a discussion of chemical methods such as proton transfer reactions, isotopic hydrogen-deuterium exchange and physical methods such as cross section measurements.

\subsubsection{Proton Transfer Reaction}

The important thermodynamic parameters of a gas-phase proton reaction are the free energy change $(\Delta \mathrm{G})$ and the enthalpy change $(\Delta \mathrm{H})$ of the reaction [37]:

$$
B+H^{+} \rightarrow B H^{+}
$$

By convention the proton affinity $(\mathrm{PA})$ is the negative of enthalpy $(-\Delta \mathrm{H})$ and gas phase basicity is the negative of the free energy change $(-\Delta \mathrm{G})$. The most direct method for obtaining GB and PA is to perform the proton transfer experiment between the protonated compound $\mathrm{B}$ and a base R of known GB. By using several reference bases the GB of the compound can be determined. 
Experimental methods have been successfully developed to account for the reactivity of singly protonated ions [38]. Measurements of the GB of charged species is much more complicated by the presence of an activation barrier to thermoneutral reactions, which arises from the separation of the two charged species. Williams and co-workers have measured the apparent gas-phase basicities of different charge states of gramicidin S [39], lysozyme [40], and cytochrome $c$ and developed a model for determining basicities of multiply charged ions [41]. By comparing calculated basicities for trial conformations from this model with the experimental thermochemistry, it is possible to propose structural information. Comparisons of calculated and experimental gas-phase basicities by Gross et al. [40] revealed that multiple structures were present within individual charge states of disulfide-intact and disulfide-reduced lysozyme ion. For the +9 and +10 charge states, the gas-phase basicities for disulfide-reduced ions were greater than values for the disulfide-intact ions. When protons were removed from high charge states of the disulfide-reduced ions, charge states below +8 were found to have similar reactivities, indicating that extended forms of disulfide-reduced ions had transformed into more compact conformers by folding in the gas phase.

McLucky and co-workers [42], reported the first proton-transfer studies for bio-molecule ions using ion trap techniques. They studied different charge states of cytochrome $c$ exposed to dimethylamine vapor in the trap. Rate constants for proton transfer were found to decrease with decreasing charge state. As charges are removed, the remaining protons will be held more strongly. Smith and co-workers have developed a ' $\mathrm{Y}$ ' tube inlet reactor, where reagents are entrained in the flow of gas in the ESI source capillary. This configuration has been used in a number of studies to investigate the transfer of protons from positively charged ions to neutral bases [43]. 
Limited experiments have been performed on oligonucleotide ion reactions. Proton transfer reactions of small multiply charged oligonucleotide anions have been studied with the ' $\mathrm{Y}$ '- tube reactor method [44] and with a quadrupole ion trap [45]. In the former case, the oligonucleotide anions were reacted with a mixture of protonated water and methanol clusters, produced by an atmospheric pressure discharge. For the latter case, protonated pyridine formed by self-chemical ionization in the ion trap served as the cationic reactant. In both cases, the major reaction was:

$(M-n H)^{n^{-}}+Y H^{+} \rightarrow(M-(n-1) H)^{(n-1)^{-}}+Y$

There was evidence for the production of a fragment ion from anions of $5^{\prime}-\mathrm{d}(\mathrm{pAAA})-3^{\prime}$ in the ' $Y$ '-tube study. No fragmentation was observed in the ion trap studies.

\subsubsection{Hydrogen-Deuterium (H/D) Exchange}

In solution the rates at which hydrogens located at peptide amide linkages in proteins undergo isotopic exchange when a protein is exposed to $\mathrm{D}_{2} \mathrm{O}$ depend on whether these amide hydrogens are hydrogen bonded or accessible to the aqueous solvent. Hence, amide hydrogen exchange rates are a sensitive probe for detecting changes in protein conformation and dynamics in solution [46].

The first applications of ESI-MS to monitor H/D exchange were reported by Katta and Chait, when they detected the exchange of labile hydrogens with deuterium by monitoring the mass increase of protein ions [47]. In the case of ubiquitin they showed that only $73 \%$ of the exchangeable hydrogens in native ubiqutin exchanged, compared to $96 \%$ exchange in the unfolded protein. This indicates the expected result for a fully denatured protein in which all exchangeable hydrogens have access to the denatured solvent, and for the folded protein in 
which some hydrogens are protected from exchange by the secondary and tertiary structure of the protein.

H/D exchange studies combined with mass spectrometry, by different groups on different proteins have provided additional information about the folding process, shedding light on the nature of transient folding intermediates undetected by NMR alone [48]. It has been also shown that several globular proteins, when placed under certain denaturing conditions exist in partially folded equilibrium states. These molten globule conformations are thought to be transient intermediates in the folding pathway, thus they have been probed by H/D exchange $[25,49]$.

Gas-phase H/D exchange of proteins was first reported by Smith and co-workers [50]. They reacted electrosprayed proteins with $\mathrm{D}_{2} \mathrm{O}$ as ions traveled through the desolvation region of an ESI-MS system. In 1993, McLafferty and co-workers [51] used FTICR techniques to investigate the conformation of five proteins. Trapped ions were exposed to dilute concentrations of $\mathrm{D}_{2} \mathrm{O}$ vapor and exchange levels were monitored for extended time periods (seconds to $30 \mathrm{~min}$.). This and similar studies have shown that there are multiple stable conformer forms of peptides and protein ions. The observation of a larger number of forms in the gas-phase has been attributed to forms that interconvert rapidly in the solution phase but not in the gas-phase, hence, becoming distinguishable forms [52].

To date, most gas-phase exchange studies with large biomolecules have been performed on positively charged protein ions. Recently researchers have begun to examine the H/D exchange of negatively charged species. Hemling et al. performed H/D exchange on nucleotides in the source region of an ESI-MS to determine the number of labile hydrogens on each molecule [53]. Robinson et al. investigated the H/D exchange kinetics of deoxynucleotide 
monophosphates with $\mathrm{D}_{2} \mathrm{O}$ [54]. Relative exchange rates were determined by monitoring the increase in mass for each sample over an extended reaction period in a FTICR mass spectrometer. They made the critical observation that 5'-monophosphate and 3'-monophosphate nucleotide anions undergo rapid H/D exchange, whereas 3'-5' cyclic monophosphate anions do not. The immediate conclusion is that the conformationally flexible phosphate group must be the initial site for H/D exchange in gas-phase nucleotide anions. They proposed a mechanism in which the phosphate group undergoes rapid exchange followed by slow intramolecular exchange with other sites within the molecule.

In another study of nucleotide H/D exchange, Freitas et al. demonstrated a dramatic increase in $\mathrm{H} / \mathrm{D}$ exchange rate of nucleotides with $\mathrm{D}_{2} \mathrm{~S}$ relative to $\mathrm{D}_{2} \mathrm{O}$ [55]. The time required to measure such rates experimentally decreased from hours for $\mathrm{D}_{2} \mathrm{O}$ to minutes for $\mathrm{D}_{2} \mathrm{~S}$ due to smaller gas-phase acidity differences between the exchange reagent and the nucleotide. Gasphase H/D exchange of oligonucleotides was also performed during ion storage in a hexapole ion reservoir by Griffey and co-workers [56]. Following exchange, the degree of H/D exchange was determined using FT-ICR mass spectrometry. It was shown that H/D exchange could be conducted more than 100 time faster than observed in conventional in-cell exchange in FTICR that requires lower gas pressures and additional pump down periods.

\subsubsection{Collision Cross Section Measurements}

Of the various methods used to study gas-phase bio-molecules, measurements of collision cross sections give the most direct insights to folding [57]. Collision cross sections are also important for understanding the transport of ions through relatively high pressure regions of the sampling interface of an ESI mass spectrometer system $[17,58]$ or the collision cell of an tandem mass spectrometer system [59]. Cross sections have been determined in ion mobility 
experiments [60], energy loss experiments [61] and energetic surface imprinting experiments [62]. Details of the theory and methods of these experiments are described in chapter 2 of this thesis. Of these methods ion mobility has the highest resolution and can, in some cases, separate ions of a biopolymer that have the same mass to charge ratio but different conformations. The energy loss method is lower in resolution, but is easily implemented on some triple quadrupole mass spectrometer systems and can be applied to fragile ions such as non-covalent complexes [63]. Neither method provides a direct measurement of the ion projection cross section. This is a predicted value based on modeled interactions between the analyte molecule and background gas. The manner in which energy and momentum transfer is modeled for a collision has a significant effect on the calculated cross section. It has been shown that cross sections determined by ion mobility and energy loss experiments agree within a few percent provided both experiments are interpreted with a collision model that corresponds to diffuse scattering [64]. Most cross section measurements to date have concerned peptide and protein ions. There has been only one report of cross sections for oligodeoxynucleotide ions [65]. In that study the cross sections of anions of the10-mer of polythymine were measured and were shown to increase with charge state in a manner similar to protein ions. Modeling showed the charges were distributed evenly along the phosphodiester backbone to minimize Coulomb repulsion.

\subsection{Goals of this research}

The advent of ESI [3] and MALDI [4] has allowed the formation of gas phase ions of intact biomolecules such as peptides, proteins and oligonucleotides. This has led to a revolution in the applications of mass spectrometry to life sciences. Studies of biomolecular ions in the gas-phase provide information about the nature of conformation in the absence of solvent, thus 
the role of intramolecular interactions and solvent can in principle be distinguished by comparing the similarities between gas and solution-phase structures.

An interesting issue in the mass spectrometry of nucleic acids is the severe fall-off in signal intensity with increasing mass, observed in some MALDI and ESI experiments [66-68]. This fall-off is not generally observed for proteins. The fall-off in signal intensity limits the analysis of dideoxy sequencing reaction products by mass spectrometry. One factor that might play a role in the phenomenon is differences in the transfer efficiency of protein and DNA ions from the ionization region to the mass analyzer. Such differences, if they exist, might stem from differences in collision cross sections. Investigating this issue was the main motivation for the present work. Studying oligonucleotide ions in order to gain structural information is currently a challenging and interesting area of mass spectrometry. This information may provide insights for DNA sequencing by mass spectrometry. 


\section{Chapter 2 Collision Cross Sections of Gas Phase Bio-}

\section{Molecules}

This chapter discusses different experimental methods and their supporting theories for collision cross section measurements of gas-phase bio-molecules.

\subsection{Introduction}

The determination of molecular structure by mass spectrometry requires that the ionized molecules undergo structurally informative fragmentation [69]. Fragmentation may be induced by the ionization process itself or by some other methods. Often the optimum methods of ion formation do not provide sufficient energy to cause fragmentation of the molecular ion. In these circumstances the internal energy of the ion can be increased by collisional activation. Tandem mass spectrometry (MS/MS) is the most important technique for acquiring collisionally activated dissociation (CAD) mass spectra. The possibility of a collision in collision cell of the mass spectrometer depends on the target gas pressure and the collision cross section of the gasphase ion [59]. Therefore measurements of collision cross sections are important in understanding the $\mathrm{CAD}$ process in tandem mass spectrometry of molecules.

Efficient coupling between an ion source working at atmospheric pressure and the high vacuum section of a mass analyzer can be achieved by use of a rf-only quadrupole operating at an intermediate pressure [17]. Ions coming from the high-pressure region of an ion source through an input orifice may possess a broad energy distribution. The rf quadrupole field keeps ions near the axis, while their axial energies are dissipated in collisions with molecules of the 
background gas [70]. Knowledge of the collision cross sections of ions being analyzed is required for modeling and improving the transport of ions through low-density gases.

Finally the collision cross section is a measure of ion "size", which can provide direct evidence for changes of higher order structures of ions of gas-phase bio-molecules like proteins and DNA. Collision theory [71] predicts a direct dependence of the collision frequency on the cross section of an ion. Thus, ions of two different sizes (i.e. different conformers) are expected to exhibit different behavior in a collision cell. Larger unfolded ions (higher charge states) will have higher collision frequencies and correspondingly will lose more axial kinetic energy than smaller folded ions [72]. Therefore, measurements that depend on the number of collisions that are experienced by different conformers can be used to discriminate between conformer shapes.

\subsection{Experimental Methods for Measuring Collision Cross Sections}

\subsubsection{Energy Loss}

Covey and Douglas [61] first described an energy loss method for the determination of collision cross sections for gas-phase protein ions with a triple quadrupole mass spectrometer. Ions from an ESI source enter a rf quadrupole $(\mathrm{Q} 0)$ at the potential of the orifice but have a sufficient number of collisions with the gas expanding from the orifice that their energies are moderated to a few electron-volts or less in $\mathrm{Q} 0$. Therefore at a collision cell (Q2) the ions appear to be formed at a potential close to the $\mathrm{Q} 0$ rod offset. Because the potential difference between the $\mathrm{Q} 0$ and the collision cell $(\mathrm{Q} 2)$ rod offset voltages was $10 \mathrm{~V}$, ions entered the collision cell with an energy of approximately $10 \mathrm{ieV}$, where $\mathrm{i}$ is the number of charges on the ion. With no collision gas added, stopping potentials at the exit of the collision cell were $10 \pm 0.5$ $\mathrm{V}$, in accord with this interpretation. Under these conditions, no collision-induced dissociation 
was seen. In Q2 the ions lose kinetic energy through collisions with the target gas. The energy of the ions leaving the cell can be determined by scanning the third quadrupole (Q3) rod offset potential. Q3 was operated in mass-analyzing mode and the rod offset voltage was increased in steps of $1.0 \mathrm{~V}$ until the ion signal was attenuated by three orders of magnitude. Stopping curves were obtained at several different cell pressures. The collision cross sections were derived by interpreting the energy loss data with a simple collision model. An important outcome of this work was that it was the first to show that collision cross sections increase with increasing charge state; the authors attributed this to increased Coulombic repulsion within the ions. Later Douglas [73] revised these values by inclusion of an aerodynamic drag coefficient. This modification showed that previously reported cross sections had been overestimated by $\sim 25 \%$.

The scattering process of the ion-neutral collision has been considered in detail by Chen et al. [64] by measuring energy losses for two proteins (cytochrome $c$ and apo-myoglobin) using different target gases The results from this study show that a diffuse scattering model provides a more accurate representation of the experimental data than a model based on specular scattering. Collings and Douglas [74] have examined the gas-phase ion structures of holo- and apo-Mb by measuring the collision cross section of each ion using the energy loss method.

\subsubsection{Simple Model}

A simple hard sphere model was used in the first determination of collision cross sections for protein ions with the energy loss method [61]. Here ions and target gas molecules were considered as hard spheres; collisions are elastic and the thermal motion of the target gas was ignored. These assumptions make this model too simplistic; however it provides a simple first estimate for determination of cross sections. In a typical inelastic collision the ratio of laboratory (lab) energy of an ion after collision with a neutral $\left(\mathrm{E}^{\prime}{ }_{\mathrm{lab}}\right)$ to that before collision 
$\left(\mathrm{E}_{\text {lab }}\right)$ is given by [75]

$$
\frac{E_{l a b}^{\prime}}{E_{\text {lab }}}=\frac{m_{1}^{2}+m_{2}^{2}}{M^{2}}-\frac{m_{2} E_{\text {int }}}{M E_{l a b}}+\frac{2 m_{1} m_{2}}{M^{2}}\left(1-\frac{E_{\text {int }} M}{E_{l a b} m_{2}}\right)^{1 / 2} \cos \theta_{c m}
$$

where $m_{1}$ is the ion mass, $m_{2}$ the neutral collision partner mass, $E_{\text {int }}$ the energy transferred to internal energy of the collision partners, $\theta_{\mathrm{cm}}$ the scattering angle in center-of-mass coordinates, and $\mathrm{M}=\mathrm{m}_{1}+\mathrm{m}_{2}$. For an elastic collision, $\mathrm{E}_{\mathrm{int}}=0$, and equation (2.1) reduces to

$$
\frac{E_{l a b}^{\prime}}{E_{l a b}}=\frac{m_{1}^{2}+m_{2}^{2}}{M^{2}}+\frac{2 m_{1} m_{2}}{M^{2}} \cos \theta_{c m}
$$

For hard-sphere collisions, $\theta_{\mathrm{cm}}$ is distributed between $0^{\circ}$ and $180^{\circ}$ with a uniform distribution. With an average energy transfer corresponding to $\theta_{\mathrm{cm}}=90^{\circ}$ equation (2.2) becomes

$$
\frac{E_{l a b}^{\prime}}{E_{l a b}}=\frac{m_{1}^{2}+m_{2}^{2}}{M^{2}}
$$

To interpret the experimental data, equation (2.3) was taken as a measure of the average energy change per collision. Given the average ratio of ion energies before and after a single collision, the energy loss in passing through a cell with several collisions could be derived.

Define $\alpha$ as the average ratio of lab energy after one collision $\left(E_{1}\right)$ to that before any collisions $\left(\mathrm{E}_{0}\right)$, given by equation $(2.3)$

$$
\alpha=\frac{E_{1}}{E_{0}}=\frac{m_{1}^{2}+m_{2}^{2}}{M^{2}}
$$

After two collisions, the average lab energy $\left(\mathrm{E}_{2}\right)$ is given by 
$E_{2}=\alpha \cdot E_{1}=\alpha^{2} \cdot E_{0}$

and after $\mathrm{N}$ collisions,

$E_{N}=\alpha^{N} \cdot E_{0}$

The total number of collisions, $\mathrm{N}$, is determined by the cross section, $\sigma$, the gas number density, $\mathrm{n}$, and the length of the collision cell, 1.

$N=\frac{l}{\lambda}=n \sigma l$

Where $\lambda$ is the mean free path. Let $\beta=1 / \alpha$, and define the target thickness $S$ by $S=n l$, then equation (2.6) gives

$\frac{E}{E_{0}}=e^{-\sigma \ln \beta}$

Equation (2.8) shows an exponential energy loss with increasing target thickness.

Experimental energy loss data can be fitted to this equation and collision cross sections can then be calculated. This model provides a simple first estimate to the energy losses of protein ions in collisions with neutrals.

\subsubsection{Drag Coefficient Model}

A bio-molecule moving through a collision gas is similar to any object moving through a gas and will always experience some resistance to its movement. The force exerted by the gas on a moving object is called the aerodynamic force. In general, the aerodynamic force on an object will depend on the size, shape, surface characteristics and velocity of the object relative 
to the gas as well as the properties of the gas itself [76]. The drag coefficient, $C_{D}$, relates the aerodynamic force on an object moving through a gas with speed $v$ to the cross sectional area, A, by following equation [77]

$$
F_{D}=m_{1} \frac{d v}{d t}=-C_{D} \frac{A n m_{2} v^{2}}{2}
$$

where $m_{1}$ is the mass of the particle, $m_{2}$ is the mass of the gas atom or molecule, $n$ is the number density, $A$ is the projection area i.e. cross section, $v$ is the speed of the particle, and $C_{D}$ is the drag coefficient. The drag coefficient $\left(C_{D}\right)$ varies with [78]:

1. Different flow regimes of gas, through the Knudsen number $K_{n}=\lambda / D$, where $D$ is the diameter of the object and $\lambda$ is the mean free path of the gas. In this study of DNA ions with energies from $50 \mathrm{eV}$ to $260 \mathrm{eV}$ entering a collision cell with a gas pressure of $\sim 1 \times 10^{-3}$ Torr, the Knudsen number is very high. The flow regime is free molecular flow.

2. The ratio of the object's speed to the thermal speed of the gas, given by

$$
s=\frac{v}{\sqrt{\frac{2 k_{B} T}{m_{2}}}}
$$

where $\mathrm{k}_{\mathrm{B}}$ is Boltzmann's constant, $\mathrm{T}$ the gas temperature and $\mathrm{m}_{2}$ is the mass of the gas atom. For the DNA ions studied s ranges from $2.98-4.68$.

3. The gas density, viscosity and the relative velocity of the flow through the Reynolds number $R=\rho v d / \eta$, where $\rho$ is the gas density, $\eta$ is the gas viscosity, $v$ is the relative velocity of the flow, and $\mathrm{d}$ is the cross-sectional diameter of the object. For the experiments discussed here the Reynolds number is almost zero.

Several groups have measured drag coefficients of small spheres in low-density gases $[77,79,80]$. The experimental drag coefficients include inelastic collisions and scattering through 
a range of angles from a rough surface. Theories have been developed to incorporate these effects in drag coefficient calculations $[81,82,83]$. The basic assumptions involved in these calculations are (1) the gas molecules have a Maxwellian velocity distribution superimposed upon a uniform flow velocity, and (2) collisions between impinging and re-emitted molecules are negligible. The type of re-emission of the molecules from the body surface can be divided into two more common categories of specular and diffuse scattering. For the case of specular scattering, the molecules leave the surface in a direction determined by the angle of incidence. Thus, the normal component of velocity is reversed, while the tangential component remains unchanged. This is the same as hard sphere scattering. For the case of diffuse scattering all memory of the incident velocity is lost. Molecules leave the surface in a cosine distribution about the normal to the surface and upon leaving the surface they will have a Maxwellian distribution of speeds, which depends on the temperature of the surface [82]. The drag coefficient can be calculated by considering the momentum transfer from incident gas striking the surface and from scattered gas leaving the surface, integrated over all scattering angles and the appropriate thermal distribution of speeds. Epstein [81] first calculated the force on a sphere in a low density gas for low speed ratios i.e. $\mathrm{s}<<1$. His results in terms of a drag coefficient defined by equation (2.9) for specular scattering is,

$$
C_{D s}=\frac{16}{3 s \sqrt{\pi}}
$$

and for diffuse scattering

$$
C_{D d}=\frac{13}{9} \frac{16}{3 s \sqrt{\pi}}
$$

Where $C_{D s}$ is the drag coefficient for specular scattering and $C_{D d}$ the drag coefficient for diffuse scattering and $s$ is the ratio of the object's speed to the thermal speed of the gas. Stadler and 
Zurick [82] calculated the drag coefficient for a sphere for the general case of all speed ratios and found, for specular scattering,

$$
C_{D s}=\frac{2}{\sqrt{\pi}} \frac{e^{-s^{2}}}{s}\left(1+\frac{1}{2 s^{2}}\right)+2\left(1+\frac{1}{s^{2}}-\frac{1}{4 s^{4}}\right) \operatorname{erf}(s)
$$

and for diffuse scattering,

$$
C_{D d}=\frac{2}{\sqrt{\pi}} \frac{e^{-s^{2}}}{s}\left(1+\frac{1}{2 s^{2}}\right)+2\left(1+\frac{1}{s^{2}}-\frac{1}{4 s^{4}}\right) \operatorname{erf}(s)+\frac{2 \sqrt{\pi}}{3 s}
$$

where erf (s) is an error function.

Henderson [83] has given a summary of all theoretical and experimental data for drag coefficients up to 1976. Diffuse scattering was in general found to give the best fit to experimental data. Drag coefficients used in this study have been calculated using equation (2.14) for diffuse scattering.

The simple hard sphere model used previously by Covey and Douglas [61], with no thermal motion of the gas, corresponds to specular scattering with $\mathrm{C}_{\mathrm{Ds}}=2.0$. In practice, the collisions between bio-molecule ions and neutral target gas may not meet some of the assumptions of the simple model. Later Douglas [73] improved the collision model by including the concept of aerodynamic drag in his model of energy loss experiments. This revised model is briefly described as follows.

Equation (2.9) can be written as:

$$
\frac{1}{v} d v=-C_{D} \frac{A n m_{2}}{2 m_{1}} d x
$$

Integrating equation (2.15),

$$
\int_{v_{0}}^{v} \frac{1}{V} d v=\int_{0}^{1}-\frac{C_{D} A n m_{2}}{2 m_{1}} d x
$$


gives

$\ln \left(\frac{v}{v_{0}}\right)=-\frac{C_{D} A n m_{2} I}{2 m_{1}}$

where $v_{0}$ is the speed of the ions entering the collision cell, $v$ is the speed of the ions leaving the cell, and 1 is the length of the collision cell. Equation (2.17) can be written as

$\ln \left(\frac{v}{v_{0}}\right)^{2}=-\frac{C_{D} A n m_{2} l}{m_{1}}$

Thus $\quad\left(\frac{v}{v_{0}}\right)^{2}=\exp \left(-\frac{C_{D} A n m_{2} l}{m_{1}}\right)$

The kinetic energy of the particle $(\mathrm{E})$ is proportional to the square of the velocity of the particle. Therefore, the energy losses of ions in the collision cell are fit to

$$
\frac{E}{E_{0}}=\exp \left(-\frac{C_{D} A n m_{2} l}{m_{1}}\right)
$$

Experimental drag coefficients include the effects of inelastic collisions, the thermal speed of the target and scattering through a range of angles from a rough surface. Therefore, the drag coefficient model is expected to provide a better description of the collisions between biomolecule ions and neutral gases.

\subsubsection{Ion Mobility}

\subsubsection{Introduction}

The mobility of a gas phase ion is a measure of how fast it moves through a buffer gas under the influence of an electric field. Ion mobility spectrometry, also called plasma chromatography, was first developed in 1970 by Cohen and Karasek [84]. In the 1970s and 1980s, several groups demonstrated that ion mobilities could be used to resolve structural 
isomers of gas phase ions [85]. In the1990's Jarrold's [86] and Bowers's [87] groups used this technique to study atomic cluster ions such as carbon clusters, and developed theoretical methods that allowed detailed structural information to be obtained. Deducing structural information from mobilities requires calculation of the average collision cross sections for trial geometries, for comparison with the experimental results. When a calculated mobility for a trial geometry is in good agreement with experiment, then this trial geometry becomes a candidate for the structure of the ion [60].

Since the development of ionization methods such as ESI and MALDI the ion mobility method has been used to examine the conformations of peptides, proteins and oligonucleotides in the gas phase. Bowers and co-workers [88] reported ion mobility measurements for ions of the peptide bradykinin, which was produced by MALDI. Jarrold, Clemmer and co-workers have performed a series of ion mobility measurements for protein ions produced by ESI. ESI generates protein ions in a distribution of charge states, so that ion mobility measurements can be performed as a function of the charge. Measurements have been performed for cytochrome $c$ [89], bovine pancreatic trypsin inhibitor (BPTI) [90], apomyoglobin [91], lysozyme [92] and ubiquitin [93]. All the above-mentioned experiments confirmed the results originally obtained by Smith and co-workers [94] and Douglas and Covey [61] that protein structure is influenced by the Coulomb repulsion energy between charges. Low charge states have tightly folded conformations that can be more compact than the crystal form of the protein, because the intramolecular binding interactions are not weakened by solvent-protein interactions. In solution, polar side chains extend out into the solvent to maximize their interactions and the protein contains cavities large enough to accommodate water molecules. As the number of charges on the gas-phase protein ion increases, more open conformations are favored. The 
unfolding transition that occurs as the charge is increased results from Coulomb repulsion. Clemmer and co-workers [95] have examined how the position of charged sites influences conformation by measuring cross sections for negatively charged cytochrome $c$ ions. Cross sections for positive and negative ions with similar number of charges are nearly identical. So far there has been only one report of ion mobility measurements of oligothymidine ions, by Clemmer's group [65]. They described cross sections of these anions and showed that cross sections increase with charge state in a manner similar to protein ions.

For calculation of ion mobility a variety of schemes have been developed ranging from a simple projection approximation to methods based on trajectory calculations. These methods are discussed briefly in following sections.

\subsubsection{Hard Sphere Model}

Ion mobility determines the drift speed of an ion in a uniform electric field [71]. The drift speed depends on the strength of the electric field, the pressure and nature of the buffer gas and the collision cross section of the ion. The apparatus for conventional mobility experiments is shown in Figure 2-1. 

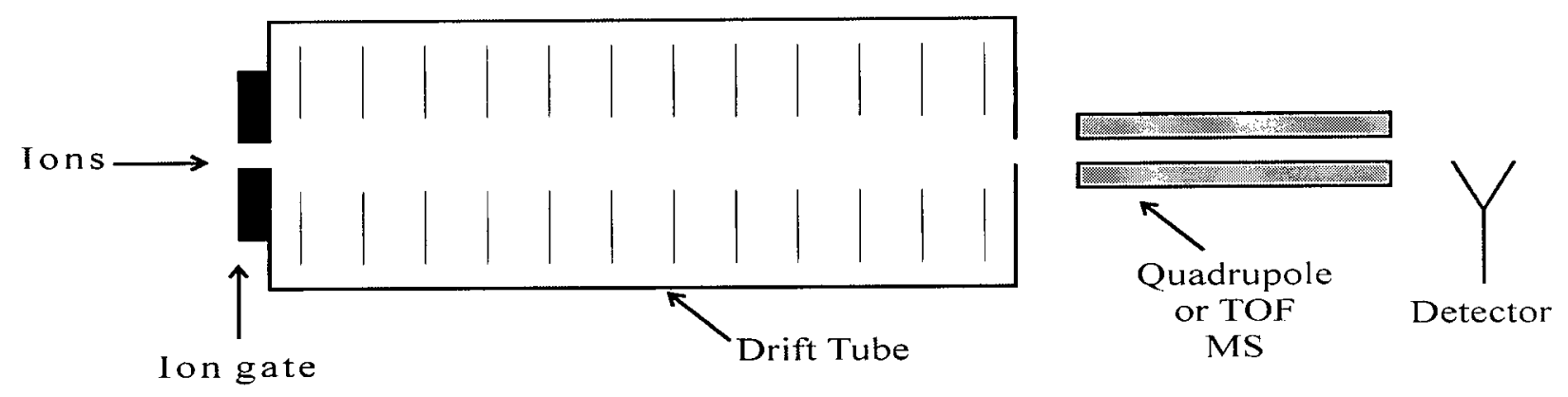

Figure 2-1. General schematic of ion mobility measurement instrument

Pulses of ion are injected through an interface into a drift tube where a uniform electric field is maintained by applying a potential gradient along a series of guard rings. The drift tube is filled with a buffer gas (helium, argon or nitrogen) at a pressure of a few Torr.

The ions drift velocities $v_{d}$ are given by:

$v_{\mathrm{d}}=\mathrm{KE}$

in which $\mathrm{E}$ is the electric field strength and $\mathrm{K}$ is the mobility. Under a given electric field the ion drift speed is constant. When ions travel through a drift tube with a length $1, \mathrm{~K}$ can be calculated from:

$K=\frac{l / t_{d}}{E}=\frac{l}{t_{d} E}$

where $t_{d}$ is the drift time.

The mobility of a given ionic species in a given gas is inversely proportional to the number density of the molecules. To facilitate the comparison and use of data, a measured mobility $\mathrm{K}$ is usually converted to a "reduced mobility", $\mathrm{K}_{0}$, defined by the equation 


$$
K_{0}=K \frac{P}{760} \frac{273.15}{T}
$$

where $\mathrm{P}$ is the gas pressure in torr and $\mathrm{T}$ is the gas temperature in Kelvin. Under the standard conditions of pressure and temperature $\left(760\right.$ torr and $\left.0^{\circ} \mathrm{C}\right)$ the gas number density $\mathrm{n}_{0}$ is $2.69 \mathrm{x}$ $10^{19} / \mathrm{cm}^{3}$, and so

$$
K_{0}=\frac{l}{t_{d} E} \frac{P}{760} \frac{273.15}{T} \quad \text { or } \quad K_{0}=\frac{l}{t_{d} E} \frac{n}{n_{0}}
$$

where $\mathrm{n}$ is the gas number density in the experiment.

For characterizing structural conformations of ions, it is useful to convert the data from mobility experiments to a collision cross section, by [71]:

$$
K=\frac{\sqrt{18 \pi}}{16}\left(\frac{1}{m_{1}}+\frac{1}{m_{2}}\right)^{\frac{1}{2}} \cdot \frac{z e}{\sqrt{k_{B} T}} \frac{1}{\Omega_{\text {avg }}^{(1,1)}} \frac{1}{n}
$$

where $m_{1}$ is the mass of the ion, $m_{2}$ is the mass of the buffer gas, ze is the charge, $n$ is the buffer gas number density, $\mathrm{k}_{\mathrm{B}}$ is the Boltzman constant, $\mathrm{T}$ is the temperature, and $\Omega^{(1,1)}$ avg is the orientationally averaged collision integral, which can be approximated by [96]:

$$
\Omega_{\text {avg }}^{(1,1)} \approx \frac{1}{8 \pi^{2}} \int_{0}^{2 \pi} d \theta \int_{0}^{\pi} d \phi \sin \phi \int_{0}^{2 \pi} d \gamma \pi b_{\min }^{2}
$$

where $b_{\min }$ is the minimum impact parameter and $\theta, \phi, \gamma$ are three angles that define the collision geometry. For a collision between two hard spheres equation (2.26) reduces to $\pi \mathrm{b}_{\min }{ }^{2}$, where $b_{\min }$ is the hard-sphere contact distance, i.e. the sum of the radii of the spheres, and so $\Omega$ equals 
the projection area A. This calculation approach is similar to a method described by Mack in 1925 in which he measured the shadow of beeswax models that were placed in different orientations with respect to a light source [97]. Now called the projection approximation method, this approach appears to provide collision cross sections for many types of geometries. However, this method ignores the potential interactions between the ion and the buffer gas and all the details of the scattering process between a polyatomic ion and the buffer gas.

Shvartsburg and Jarrold have developed a method that includes the contribution of the scattering angle to the cross section, which is referred to as the exact hard-sphere scattering (EHSS) method [98]. In this method the averaged collision integral is solved numerically.

$$
\Omega_{a v g}^{(1,1)}=\frac{1}{4 \pi^{2}} \int_{0}^{2 \pi} d \theta \int_{0}^{\pi} d \phi \sin \phi \int_{0}^{2 \pi} d \gamma \times \int d b 2 b(1-\cos \chi(\theta, \phi, \gamma, b))
$$

The scattering angle, $\chi(\theta, \phi, \gamma, \mathrm{b})$, is determined by following a trajectory through any and all of its collisions with the polyatomic ion. This model considers the details of the collision process, however, the effect of the long-range potentials has not been taken into account in this approach.

Mesleh et al. [99] have developed a "trajectory method" which includes the contribution from the long-range interactions. In this approach, atoms interact with the buffer gas along realistic potential surfaces and the dynamics of buffer gas scattering are included in the calculation.

Collision cross sections for BPTI, cytochrome $c$ and carbon clusters have been calculated using the trajectory method, EHSS and projection approximation method. The values determined using the trajectory method are up to $20 \%$ smaller than those obtained from the 
projection approximation method. The results from the EHSS method are within a few percent of those obtained from trajectory method calculations. All methods described here have assumed a rigid geometry and elastic collisions. Inelastic collisions have not been considered, which may contribute a substantial error in calculations of cross sections for polyatomic ions. In mobility experiments collisions are elastic on average.

\subsubsection{Drag Coefficient Model}

The drag coefficient could also be included in ion mobility calculations, since ions travel through a low density gas and they will experience the drag aerodynamic force. In mobility experiments, the speed ratios are very low, typically $s<<1$. Therefore, the drag coefficients can be written [64]:

$C_{D}=\frac{c}{s}$

where $\mathrm{c}$ is determined by the model used to calculate the drag coefficient. Values for $\mathrm{c}$ for the models discussed here are shown in Table 2-1. These were determined by expanding the expressions for $C_{D}$ in powers of $s$ and keeping only the coefficient of the term in $1 / s[81,82,71]$.

Table 2-1. Coefficients c in Equation (2.28)

\begin{tabular}{lllll}
\hline Author & Reference & Specular & Diffuse & Specular/Diffuse \\
\hline Epstein & 81 & 3.01 & 4.35 & 0.69 \\
Stadler \& Zurick & 82 & 3.39 & 4.56 & 0.74 \\
Mobility Theory & 71 & 3.01 & & \\
\hline
\end{tabular}

In ion mobility experiments, the drag force on an ion is balanced by the electric force $\mathrm{qE}$ ( $\mathrm{q}$ is the charge on the ion, $\mathrm{q}=\mathrm{ze}$ ) so that

$$
q E=\frac{C_{D} A n m_{2} v_{d}^{2}}{2}
$$


Substituting $C_{D}$ from equation (2.28) and s from equation (2.10)

$v_{d}=\frac{\sqrt{2}}{c} \frac{1}{\sqrt{m_{2}}} q E \frac{1}{\sqrt{k_{B} T}} \frac{1}{n} \frac{1}{A}$

where $v_{d}$ is the ion drift speed. For comparison, mobility theory, which calculates the velocity distribution for the ion, not just the drift speed, gives

$v_{d}=\frac{\sqrt{18 \pi}}{16}\left(\frac{1}{m_{1}}+\frac{1}{m_{2}}\right)^{\frac{1}{2}} q E \frac{1}{\sqrt{k_{B} T}} \frac{1}{n} \frac{1}{\Omega_{\text {avg }}^{(1,1)}}$

where $\Omega$ is the average collision integral [71]. For collisions of hard spheres $\Omega=\pi \mathrm{b}^{2}$, where b is the hard-sphere contact distance, i.e. the sum of the radii of the spheres, and so $\Omega$ equals the projection area A. For the case where $m_{1} \gg>m_{2}$ (bio-molecule ions) the mobility formula for hard spheres gives the same drift speed as that derived for the specular scattering in drag coefficient theory. For diffuse scattering the value of $\mathrm{c}$ in equation (2.28) is increased, and the drift speed for a given projection area $\mathrm{A}$ is decreased. The magnitude of decrease is given by the ratio of the factors $\mathrm{c}$ in Table 2-1. Therefore, if the diffuse scattering applies to bio-molecule ions, using the Stadler and Zurick calculations the average collision integral determined from the mobility equation (2.31) should be reduced by 0.74 , to give the projection area $\mathrm{A}$.

\subsubsection{Energetic Surface Imprinting}

In the hopes of providing further complementary information on the detailed structure of gas-phase bio-molecular ions, Reimann and co-workers [100] have exploited a visually oriented approach. Using the "multiply charged macromolecular accelerator" (MUMMA), protein ions produced by ESI are extracted into a vacuum chamber. Ions of a specific $\mathrm{m} / \mathrm{z}$ ratio are selected in a quadrupole mass filter to form an ion beam and are accelerated to impact a target at a selected total kinetic energy of around $100-300 \mathrm{KeV}$ as shown in Figure 2-2. 


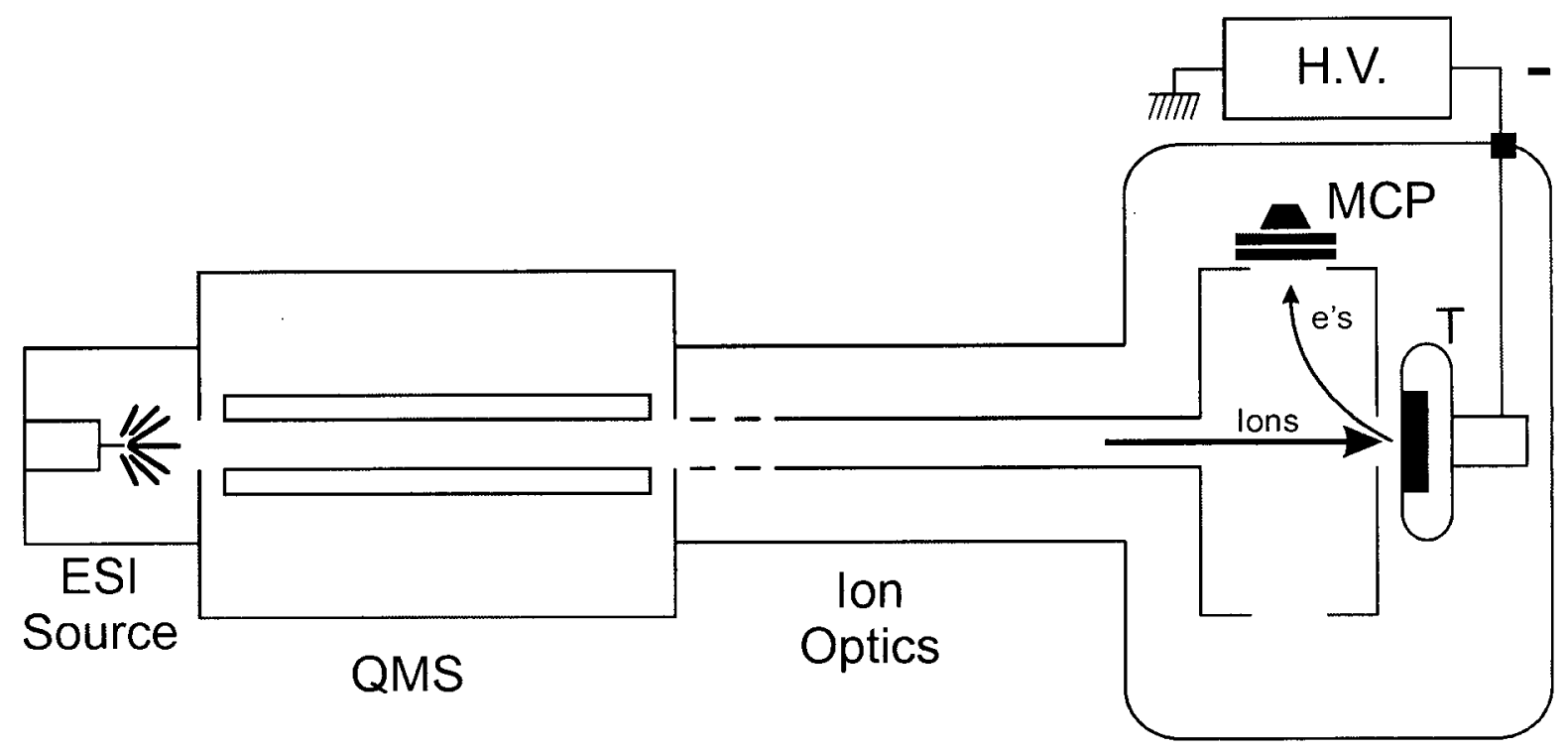

Figure 2-2 Schematic diagram of MUMMA. ESI=electrospray ionization; QMS=quadrupole mass spectrometer; $\mathrm{H} . \mathrm{V}$. = high voltage; $\mathrm{MCP}=$ multichannelplate detector; $\mathrm{T}=$ target.

Target surfaces are usually made of highly oriented pyrolytic graphite (HOPG), $\mathrm{SiO}_{2}$ or mica. Impact rates are typically $10^{3}-10^{5}$ ions per second; surfaces are irradiated with the beam for several hours. As each incident ion penetrates and collides with the graphite surface, the energy deposited diffuses and dissipates, resulting in a surface defect, which can be measured by scanning probe microscopy (SPM). The impact of highly charged biomolecules with a smooth graphite surface is sufficiently energetic that the graphite surface melts and is immediately quenched into an amorphous state. The resolidified area of impact appears as a hillock in microscopy analysis. The shape of each defect reflects the conformation and orientation of the incident ion, which created it.

Energy diffusion and surface modification lead to a broadening of the surface defects. If this broadening can be subtracted, higher resolution information on the region impacted by the protein ion and therefore on the conformation of the protein ion itself can be obtained. Because 
a thermally activated process causes the surface defects and since HOPG is highly anisotropic, a two-dimensional heat diffusion equation is employed to model this process [101]. By doing so, $L^{\prime}$ the projected length of the protein, which caused the given surface defect, can be calculated. The impact of the molecule with the surface is assumed to deposit energy along a line of length $L^{\prime}$. The length $L^{\prime}$ is taken to be the projection length of the molecule, $L$, on the surface $\left(L^{\prime}=L\right.$ $\sin \theta)$ where $\theta$ is the polar orientation angle. The molecular length $(L)$ is found from the length of the largest defect $\left(L^{\prime}\right)$.

In the first study of biomolecular ions using this method, Reimann and co-workers [100] examined the +41 charge state of disulfide-intact and +47 charge state of disulfide-reduced bovine albumin. The elliptical defects observed for the disulfide-intact ions were larger than would be expected if albumin retained its native shape; the expansion of these ions was attributed to Coulombic repulsion. The disulfide-reduced ions yielded hillocks having a wide range of sizes; large defects were assigned to the impact of the denatured ion, and smaller features were attributed to fragmentation of the ion with residual gas. Quist et al. [102] have used MUMMA to study bovine insulin (+5), bovine trypsin $(+15)$, horse heart cytochrome c $(+10)$, and bovine albumin $(+42)$ proteins. Reimann and collaborators examined surface impact sites formed by apomyoglobin and disulfide-intact and reduced lysozyme [62,101 ]. The studies of lysozyme showed that the distributions of hillock lengths produced from disulfide-reduced +10 ion shifted to higher values and were twice as broad as those observed for disulfide-intact +9 ions.

The results obtained from energetic impact imprinting studies for different charge states of apomyoglobin and lysozyme ions are in good agreement with the conclusions drawn from ion mobility [92] experiments. 


\section{Chapter 3 Instrumentation, Experimental Procedures and}

\section{Sample Preparation}

This chapter describes the experimental apparatus, sample preparation and experimental procedures used in this study. It includes an overview of the quadrupole mass filter principles, a description of the triple quadrupole mass spectrometer, the reduced liquid flow rate ESI source and ion detection. The preparation of protein and DNA solutions and other relevant reagents is also described. The experimental procedure section describes the method for ion energy loss measurements and data analysis.

\subsection{Electrospray Ionization Triple Quadrupole Mass Spectrometer}

\subsubsection{Operation Principles of Quadrupole Mass Filters}

A quadrupole field is produced by a set of four parallel hyperbolic rods. Many mass filters employ circular rods instead of hyperbolic rods due to the difficulty of manufacturing hyperbolic rods $[103,104]$. A constant direct current (dc) voltage $U$ and a radio frequency (rf) voltage Vcos $\omega t$ are applied to rods as shown in Figure 3-1. 


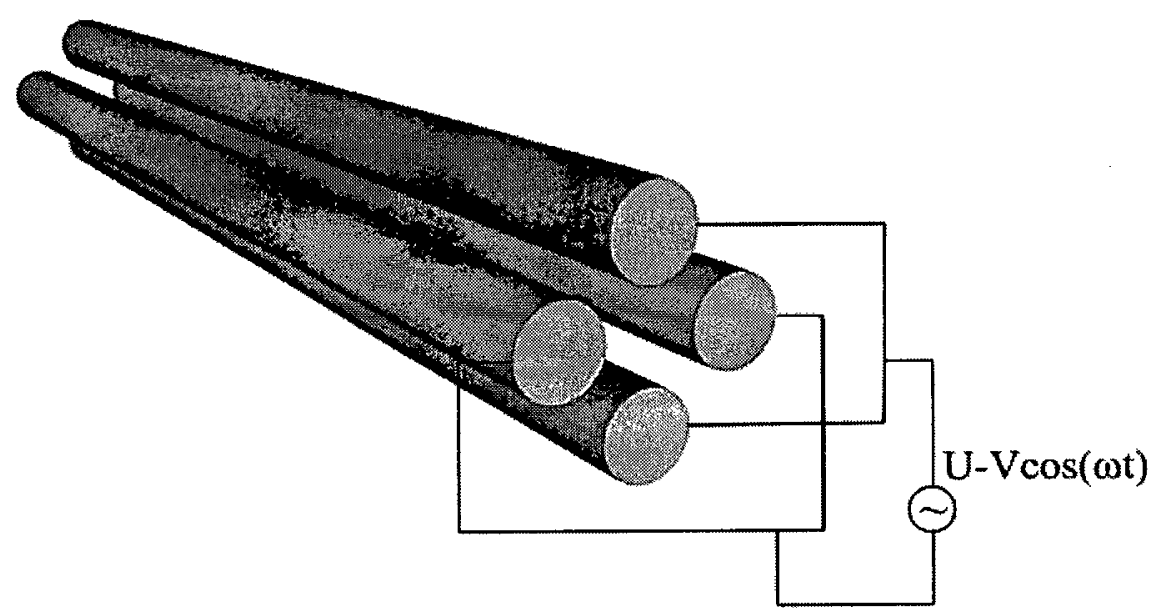

Figure 3-1 Conventional circular quadrupole rods in a quadrupole mass filter.

The potential $\Phi(\mathrm{x}, \mathrm{y})$ of any point inside the quadrupole is described by:

$\Phi(x, y)=\frac{x^{2}-y^{2}}{r_{0}^{2}} \Phi_{0}$

where $r_{0}$ the field radius, is the distance from the centre of the quadrupole to any rod. The potential $\Phi_{0}$ is given by:

$\Phi_{0}=(U-V \cos \omega t)$

where $\mathrm{U}$ is the dc voltage and $\mathrm{V}$ is the zero-to-peak amplitude of the rf voltage applied from each quadrupole rod to ground, and $\omega$ is the angular frequency of the rf voltage.

The ion motion in the $\mathrm{x}$ and $\mathrm{y}$ directions in the quadrupole field are independent and are determined by the following equations:

$$
\begin{aligned}
& \ddot{x}+\frac{z e}{m r_{0}^{2}}(U-V \cos \omega t) x=0 \\
& \ddot{y}-\frac{z e}{m r_{0}^{2}}(U-V \cos \omega t) y=0
\end{aligned}
$$


where $\ddot{x}=\frac{d^{2} x}{d t^{2}}$ and $\ddot{y}=\frac{d^{2} y}{d t^{2}}, \mathrm{z}$ is the number of charges in an ion, $\mathrm{e}$ is the electron charge and $\mathrm{m}$ is the ion mass. These differential equations can be written with dimensionless parameters, and are then the Mathieu equation:

$\frac{d^{2} u}{d \xi^{2}}+\left(a_{u}-2 q_{u} \cos 2 \xi\right) u=0 \quad \xi=\frac{\omega t}{2} \quad \mathrm{u}=\mathrm{x}$ or $\mathrm{y}$

By comparison with equation (3.3) a and q can be written:

$a_{x}=-a_{y}=\frac{8 e U}{m r_{0}^{2} \omega^{2}} \quad q_{x}=-q_{y}=\frac{4 e V}{m r_{0}^{2} \omega^{2}}$

The solution to the Mathieu equation is complex; for the purpose of this study it is sufficient to say that the Mathieu equation has two types of solution. The first is stable motion, where ions oscillate in the $\mathrm{x}-\mathrm{y}$ plane with limited amplitudes. Ions pass through the quadrupole field without hitting the rods. The second is unstable motion, where the amplitudes grow exponentially in the $\mathrm{x}, \mathrm{y}$ or in both directions, and ions are lost. The stability depends only on the $a$ and $q$ parameters. Therefore in an $a-q$ plane there are regions of stability and instability. The first overlapping region for combined $\mathrm{x}$ and $\mathrm{y}$ stability is plotted in Figure 3-2.

For fixed values of $r_{0}, \omega, U$ and $V$, all ions with the same $\mathrm{m} / \mathrm{e}$ have the same operating point in the stability diagram. Since $\mathrm{a} / \mathrm{q}$ is equal to $2 \mathrm{U} / \mathrm{V}$ and does not depend on $\mathrm{m}$, all masses lie along the operating line $\mathrm{a} / \mathrm{q}=$ constant as shown in Figure 3-2. By scanning the $\mathrm{U}$ and $\mathrm{V}$ values along this line, ions with different masses will be transmitted in sequence of mass-tocharge ratio and will reach the detector in order, producing a mass spectrum. 


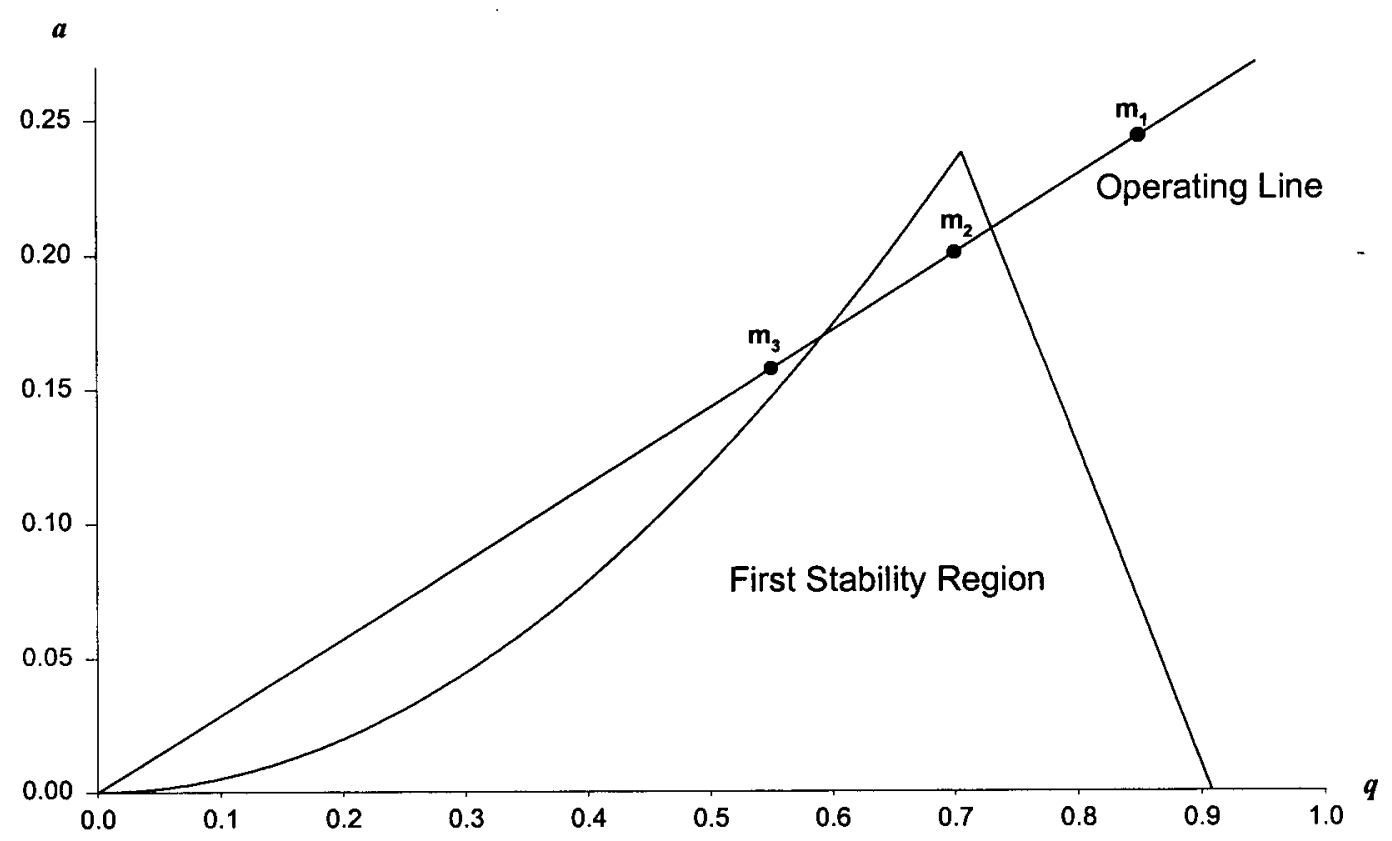

Figure 3-2. The first stability region of a quadrupole field. $m_{1}, m_{2}$ and $m_{3}$ represents ions with different masses on an operating line.

\subsubsection{Electrospray Ionization Triple Quadrupole Mass Spectrometer}

The ESI-triple quadrupole mass spectrometer used in this research is a prototype of the

PE-Sciex API-300. This instrument is shown schematically in Figure 3-3. 


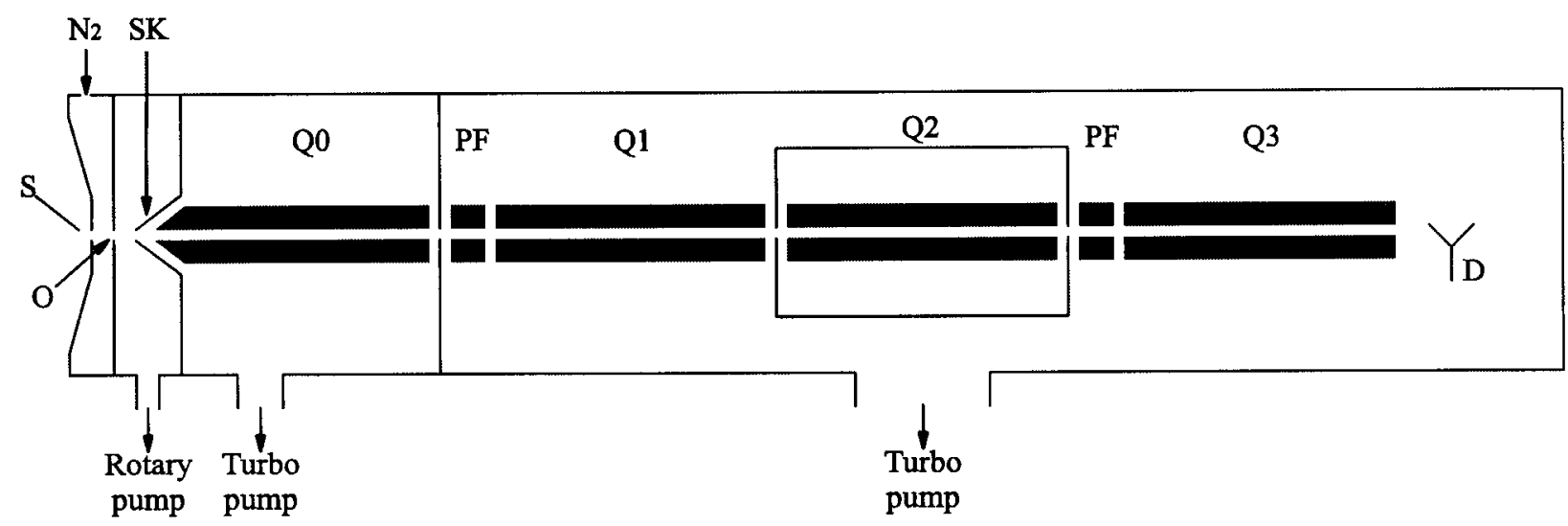

Figure 3-3 Schematic of the ESI-triple quadrupole mass spectrometer system: S, electrospray ionization source; $\mathrm{N}_{2}$, nitrogen curtain gas; $\mathrm{C}$, curtain plate; $\mathrm{O}$, sampling orifice; $\mathrm{SK}$, skimmer; Q0, rf only quadrupole; PF, prefilter; Q1, mass-analyzing quadrupole; Q2, collision cell; Q3, mass-analyzing quadrupole; $\mathrm{D}$, detector.

Ions formed by a reduced liquid flow rate electrospray source, pass through a dry nitrogen curtain gas, a sampling orifice (diameter $0.25-\mathrm{mm}$ ) into a region with a background pressure of about 2 Torr. Gas and ions enter this region in a free jet expansion, and ions can then be sampled by a skimmer (diameter $0.75-\mathrm{mm}$ ) into a rf-only quadrupole (Q0) operating at a pressure of $7 \times 10^{-3}$ Torr. Ions are collisionally cooled to an energy spread of about $1 \mathrm{eV}$. They then enter the main chamber, which includes three quadrupoles Q1, Q2 ,Q3 and two prefilters. Quadrupoles Q1 and Q3 are mass analyzing quadrupoles and Q2 is an rf-only quadrupole collision cell. The pressure in the main chamber is about $7 \times 10^{-6}$ Torr, the collision cell pressure was varied up to $1.4 \times 10^{-3}$ Torr. This apparatus has a three-stage differential pumping system. A mechanical pump is used for the interface region and turbo molecular pumps evacuate the Q0 chamber and the main chamber. Mass calibration was performed using poly propylene glycol ions and confirmed by the mass measurement of reserpine $\left(\mathrm{MH}^{+}, 609.7\right)$. 


\subsubsection{Reduced Liquid Flow Rate Electrospray Source}

This home-built electrospray source is shown in Figure 3-4.

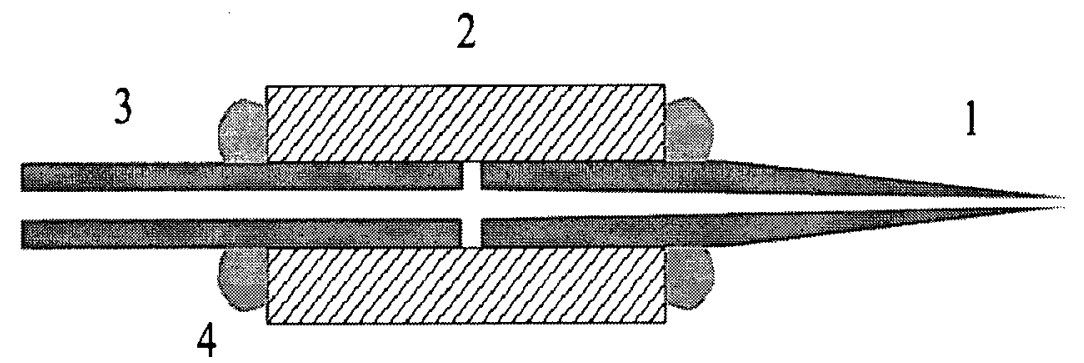

Figure 3-4. Schematic diagram of reduced liquid flow rate ESI source. 1- tapered tip; 2stainless steel junction; 3- fused silica capillary; 4- epoxy glue.

The electrospray capillary was made with a 50-cm long fused-silica capillary having a 50- $\mu \mathrm{m}$ internal diameter and 150- $\mu \mathrm{m}$ external diameter (Polymicro Technologies, Phoenix, AZ). This capillary was connected with a $4 \mathrm{~cm}$ tapered tip, inside a $2 \mathrm{~cm}$ long piece of stainless steel syringe tube with an inner diameter of $0.018-\mathrm{cm}$ and outer diameter of 0.036- $\mathrm{cm}$, (Small Parts Inc., Miami Lakes, FL). The junction was held together and sealed with epoxy glue. The tapered spray tips were pulled in-house and had an internal diameter at the tip of $\sim 20 \mu \mathrm{m}[105]$. A syringe pump (model 22, Harvard Apparatus, Saint-Laurent, QU.) pumped the solution through the fused-silica capillary to the tip, with a typical flow rate of $0.4-0.6 \mu \mathrm{l} / \mathrm{min}$.

\subsubsection{Ion Detection}

A channel electron multiplier (CEM) is used as the ion detector. The CEM is a continuous dynode device, which can detect either positive or negative ions. For positive ion mode the input is generally at a high negative potential and the output is at ground. For detection of negative ions, the input is generally at ground or some positive potential and the 
output is at a high positive voltage. An ion striking the input face of the CEM starts an electron cascade, resulting in a current pulse $\left(\sim 10^{-5} \mathrm{~A}\right)$, which is amplified. A discriminator is used to block the noise and only transmit the greater ion signals. The ion signal is recorded in ion counting mode. The recorded data are the total number of ions at different $\mathrm{m} / \mathrm{z}$ ratios. The computer calculates the total ion counts per second for each scan and these data are used to construct a mass spectrum over a certain $\mathrm{m} / \mathrm{z}$ range. In this study for detection of negative ions, the CEM electric circuit was modified as is shown in Figure 3-5.

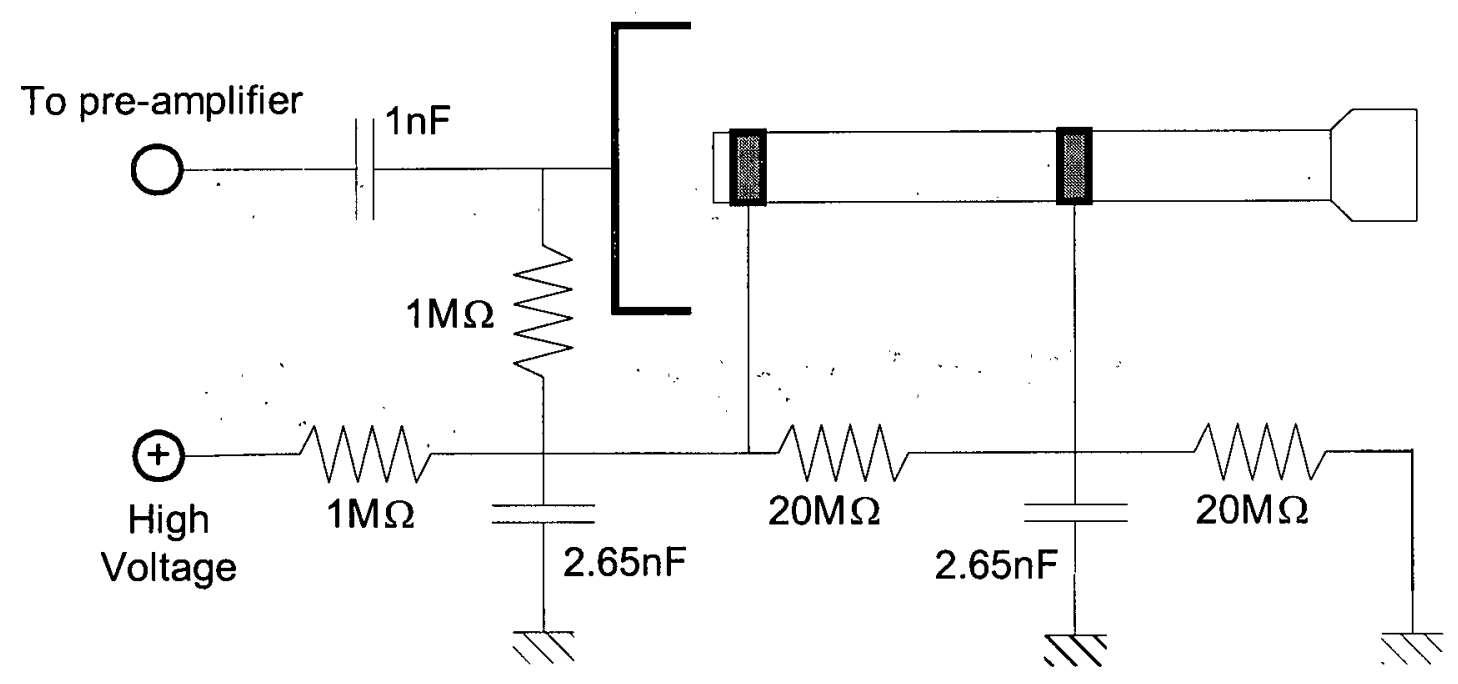

Figure 3-5 Ion detection circuit for negative ions.

\subsection{Sample Preparation}

\subsubsection{DNA and Protein Solutions}

The DNA samples were provided by the lab of Dr. Lloyd Smith, Chemistry Department University of Wisconsin. The sequence and molecular weights of the single stranded DNA samples used in this study are shown in Table 3-1. These sequences correspond to a region in the cloning vector M13mp18 [106]. All DNA samples obtained were previously purified by 
reverse-phase HPLC from Integrated DNA Technologies, Inc. (Coralville, IA) and quantified by UV absorbance at $260 \mathrm{~nm}$. The oligodeoxynucleotides were diluted in a buffer of 1:1 $\mathrm{H}_{2} \mathrm{O}: \mathrm{MeOH}, 400 \mathrm{mM}$ 1,1,1,3,3,3,-hexafluoro-2-propanol (HFIP) 99.8\% purity from Aldrich Chemical Company (adjusted to $\mathrm{pH} 7$ with triethylamine) to a concentration of $20 \mu \mathrm{m}$. Bovine red cell ubiquitin (76 residues, MW 8565), horse heart cytochrome $c$ (104 residues, MW 12,318) and horse heart holomyoglobin (153 residues, MW 17,568) were from Sigma Chemical Company (St. Louis, MO). Protein solutions were: ubiquitin, $50 \mu \mathrm{m}$ in $49 \%$ $\mathrm{CH}_{3} \mathrm{CN}, 49 \% \mathrm{H}_{2} \mathrm{O}, 2 \%$ acetic acid; cytochrome $c, 10 \mu \mathrm{m}$ in $50 \% \mathrm{CH}_{3} \mathrm{CN}, 50 \% \mathrm{H}_{2} \mathrm{O}$ adjusted to pH 3 with hydrochloric acid; myoglobin, $10 \mu \mathrm{m}$ in $50 \% \mathrm{CH}_{3} \mathrm{CN}, 50 \% \mathrm{H}_{2} \mathrm{O}, 0.1 \%$ acetic acid.

Table 3-1 DNA sequences and molecular weights

\begin{tabular}{llc}
\hline Sequence & MW \\
\hline 28-mer & 5'-TGT AAA ACG ACG GCC AGT GCC AAG CTT G-3' & $8,622.6$ \\
40-mer & 5'-TGT AAA ACG ACG GCC AGT GCC AAG CTT GCA TGC CTG & $12,331.0$ \\
& CAG G-3' & \\
55-mer & 5'-TGT AAA ACG ACG GCC AGT GCC AAG CTT GCA TGC CTG & $16,985.0$ \\
& CAG GTC GAC TCT AGA TTA A-3' & \\
\hline
\end{tabular}

\subsubsection{Other Reagents}

HPLC grade acetonitrile, methanol, acetic acid and hydrochloric acid were from Fisher Chemical Company (Nepean, ON). UHP grade nitrogen, manufacturers' stated purity (99.999\%) and Linde grade argon, manufacturers' stated purity (99.999\%), were from Praxair (Mississauga, ON, Canada). 


\subsection{Experimental Procedures}

\subsubsection{Measurements of Ion Energy Loss}

All measurements were performed with the triple quadrupole mass spectrometer described in section 3.1.2. The orifice-skimmer voltage difference was $80 \mathrm{~V}$ for DNA samples and 110 for protein samples. Ions enter Q2 with kinetic energies determined by the difference between Q0 and Q2 rod offset voltages, which was $10 \mathrm{~V}$ in this case. Therefore ions enter the collision cell with energy of approximately $10 \mathrm{eV}$ per charge. At this collision energy, no fragmentation of the ions was observed. The cell argon pressure was typically varied up to $1.4 \mathrm{x}$ $10^{-3}$ Torr, measured with a precision capacitance manometer (MKS Baratron type 120 high accuracy pressure transducer, Boulder, $\mathrm{CO}$, manufacturer's stated accuracy $0.12 \%$ ). Quadrupole Q3 was operated in mass-resolving mode. Energy distributions of ions leaving Q2 were determined from stopping curves obtained by increasing the Q3 rod offset voltage in steps of 1 $\mathrm{V}$ until the signal was attenuated by at least two orders of magnitude. Stopping curves were obtained for several different cell pressures. With no collision gas added, stopping potentials were typically $10 \pm 0.5 \mathrm{~V}$, in accord with ion energies of $10 \mathrm{eV}$ per charge. The collision cell length was $20.6 \mathrm{~cm}$. The typical potentials applied to the system are shown in Table 2-2. 
Table 3-2 Voltages used in Energy Loss Experiments

\begin{tabular}{|c|c|c|}
\hline Instrument & Voltage (V) & Voltage (V) \\
\hline Component & Negative ion mode & Positive ion mode \\
\hline Sprayer needle & -3000 & 5000 \\
\hline Curtain plate & -1130 & 1000 \\
\hline Orifice & -190 & 220 \\
\hline Skimmer & -110 & 110 \\
\hline Q0 rod offset & -103 & 103 \\
\hline Prefilter 1 & -92 & 90 \\
\hline Q1 rod offset & -88 & 100 \\
\hline Q1/Q2 lens & -57 & 80 \\
\hline Q2 rod offset & -93 & 93 \\
\hline $\mathrm{Q} 2 / \mathrm{Q} 3$ lens & -90 & 79 \\
\hline Prefilter 2 & -67 & 77 \\
\hline Q3 rod offset & $\begin{array}{c}\text { Varied } \\
(-93 \ldots-104)\end{array}$ & $\begin{array}{c}\text { Varied } \\
(93 \ldots 104)\end{array}$ \\
\hline
\end{tabular}

\subsubsection{Data Analysis of Energy Loss Experiments}

For interpreting the experimental data, the stopping potential at which the ion intensity was dropped to one-tenth of its original value with no gas in the collision cell, was taken as a measure of the ion energy. This value was interpolated from the stopping curves, which are the plot of the peak intensity vs. the potential difference between Q3 and Q2, and is referred to as $\mathrm{E}_{1 / 10}$. The validity of this procedure has been demonstrated previously by comparing the cross 
section data derived from this procedure and a Monte Carlo simulation of the experiment [61]. An example of the data analysis of the cross section measurements is given here. The stopping curves for 28-mer oligodeoxynucleotide -5 ions at cell pressures of $0,0.275,0.5805,0.853$, 1.141 mTorr of Ar are shown in Figure 3-6. Values of the stopping potentials $\left(E_{1 / 10}\right)$ from the stopping curves were $10.05,8.65,7.45,6.54$ and $5.68 \mathrm{~V}$ at those pressures respectively. Taking the stopping potential with no collision gas as $\mathrm{E}_{0}$, i.e. $\mathrm{E}_{0}=10.05 \mathrm{~V}$, the $\mathrm{E} / \mathrm{E}_{0}$ ratios can be calculated accordingly. The drag coefficient for diffuse scattering, $C_{D}$, was calculated from Equation (2.14) and all data were fit to following equation as is shown in Figure 3-7.

$$
\frac{E}{E_{0}}=\exp \left(-\frac{C_{D d} n m_{2} I A}{m_{1}}\right)
$$

The collision cross section of 28 -mer oligodeoxynucleotide $(-5)$ ion is $605 \AA^{2}$ with a very good correlation coefficient.

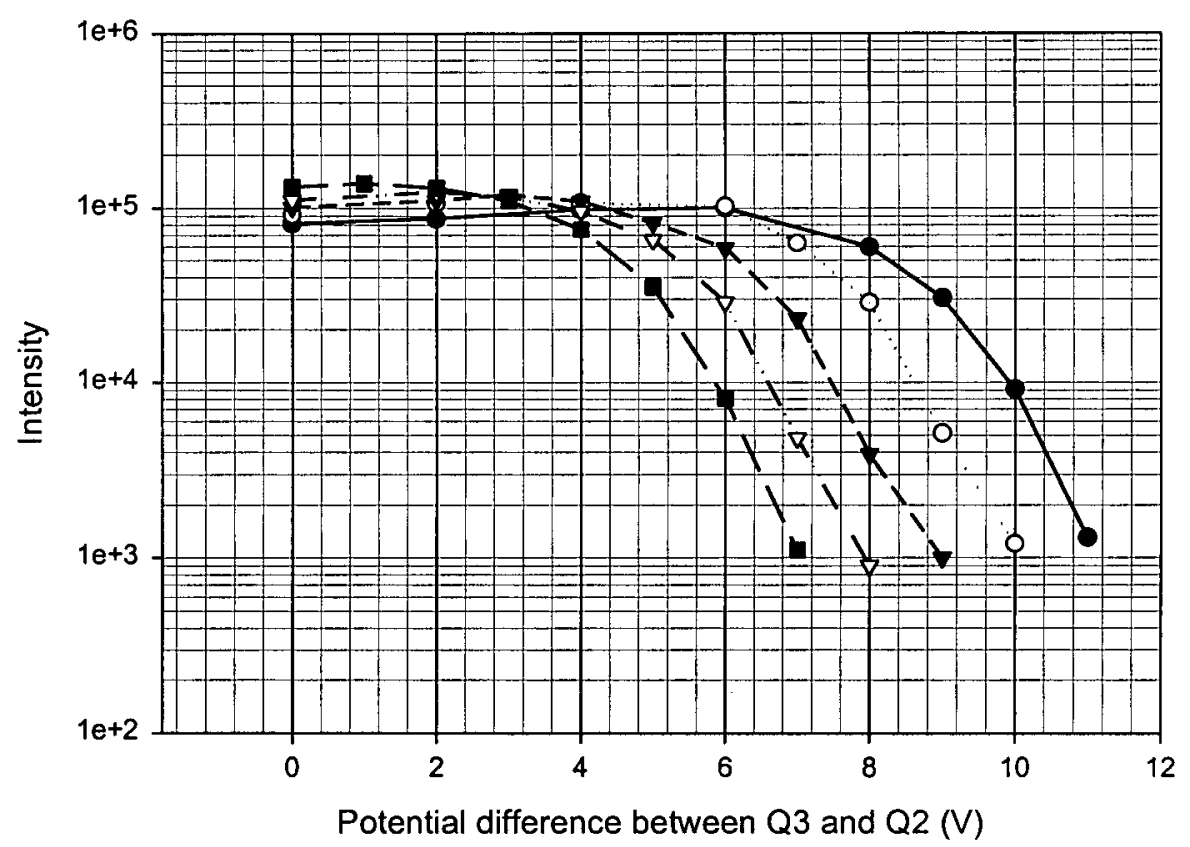

Figure 3-6. The stopping curves of 28-mer (-5) ions at different cell pressures of Ar. The curves from right to left correspond to cell pressures of $0,0.275,0.581,0.853$ and 1.141 militorr of Ar, respectively. " 0 " means no added collision gas. 


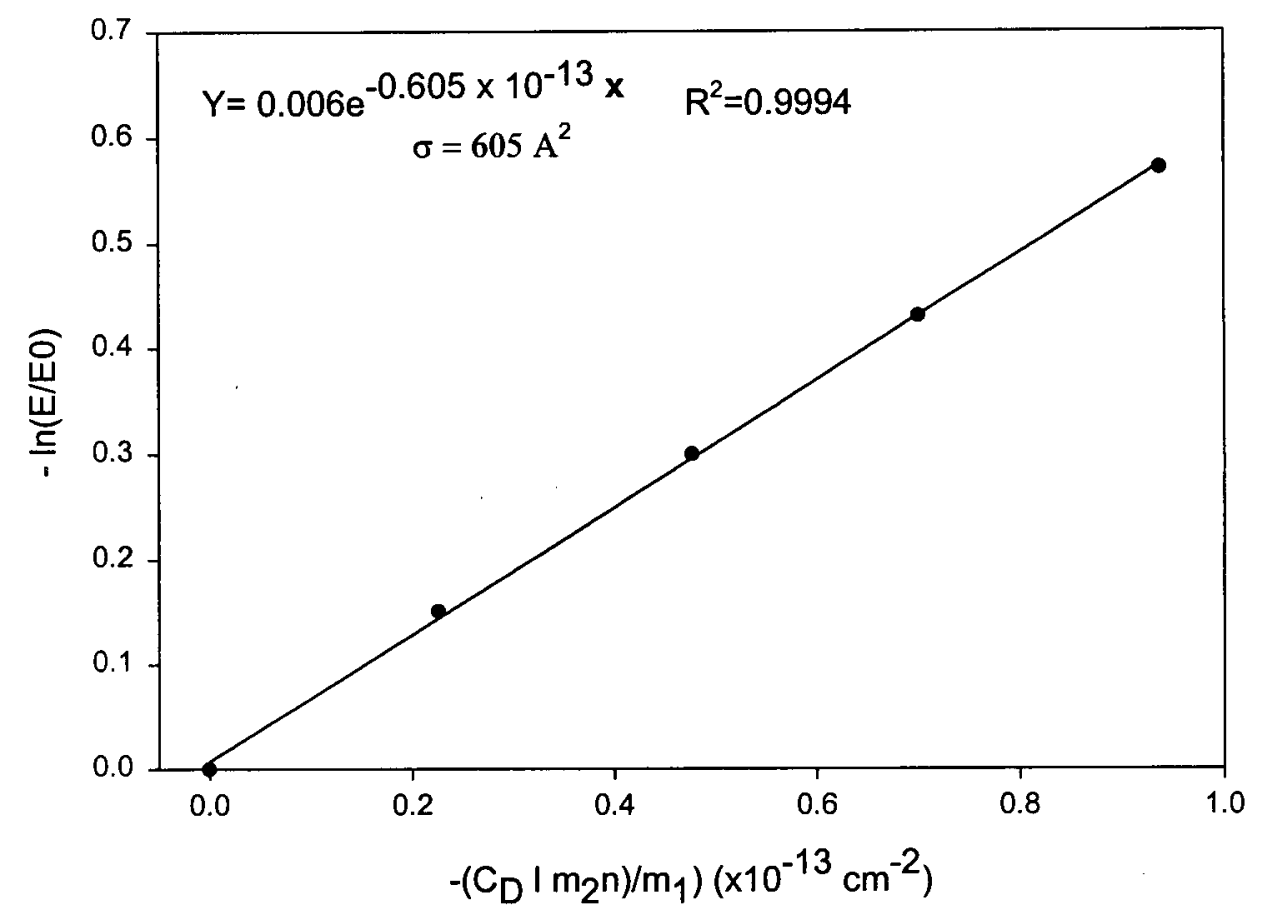

Figure 3-7. A plot of $E / E_{0}$ vs. $-\left(C_{D} 1 m_{2} n\right) / m_{1}$ for $28-m e r(-5)$ ion. 


\section{Chapter 4 Collision Cross Sections of DNA and Protein}

\section{Ions}

This chapter discusses the experimental results of cross section measurements of DNA and protein ions. Collision cross sections of negative ions of a 28-, 40- and 55-mer of single stranded DNA were measured by the energy loss method as described in chapter 3 , and compared to collision cross sections of proteins of nearly the same molecular weightubiquitin, cytochrome $\mathrm{c}$ and apomyoglobin, respectively.

\subsection{Mass spectra and sensitivities of 28, 40 and 55-mer Oligodeoxynucleotides}

\subsubsection{Mass spectra of 28-, 40 and 55-mer oligodeoxynucleotides}

Negative ion ESI mass spectra of 28-, 40- and 55-mer oligodeoxynucleotides at an

orifice-skimmer voltage difference of $80 \mathrm{~V}$ are shown in Figure 4-1, 4-2 and 4-3, respectively.

Under the experimental conditions used (chapter 3) oligodeoxynucleotides ions produced highly charged ions. For the 28-mer oligodeoxynucleotide (M.W. 8,622.6) eight charge states, $\{-5\}$ to $\{-12\}$ were produced corresponding to $\mathrm{m} / \mathrm{z}: 1722\{-5\}, 1435\{-6\}, 1231\{-7\}, 1077\{-8\}, 957\{-$

$9\}, 861\{-10\}, 782\{-11\}$, and $717\{-12\}$. For the 40-mer oligodeoxynucleotide (M.W.

12,331.0) eleven charge states, $\{-7\}$ to $\{-17\}$ were produced corresponding to $\mathrm{m} / \mathrm{z}: 1759\{-7\}$, $1540\{-8\}, 1369\{-9\}, 1232\{-10\}, 1120\{-11\}, 1026\{-12\}, 947\{-13\}, 879\{-14\}, 821\{-15\}$, $769\{-16\}$, and $724\{-17\}$. For the 55-mer oligodeoxynucleotide (M.W. 16,985.0) twelve charge states, $\{-15\}$ to $\{-26\}$ were produced corresponding to $\mathrm{m} / \mathrm{z}: 1128\{-15\}, 1057\{-16\}, 995\{-17\}$, 
$940\{-18\}, 890\{-19\}, 845\{-20\}, 805\{-21\}, 769\{-22\}, 735\{-23\}, 705\{-24\}, 676\{-25\}$ and $650\{-26\}$. It is well established that native conformations of proteins in solution produce low charge states in ESI and high charge states of proteins are usually formed when a protein unfolds in solution [20]. Little work has been done to investigate such relationships for nucleic acids. The high charge states observed for these DNA samples suggest that they have loose unfolded conformations in solution, comparable to those of denatured proteins.

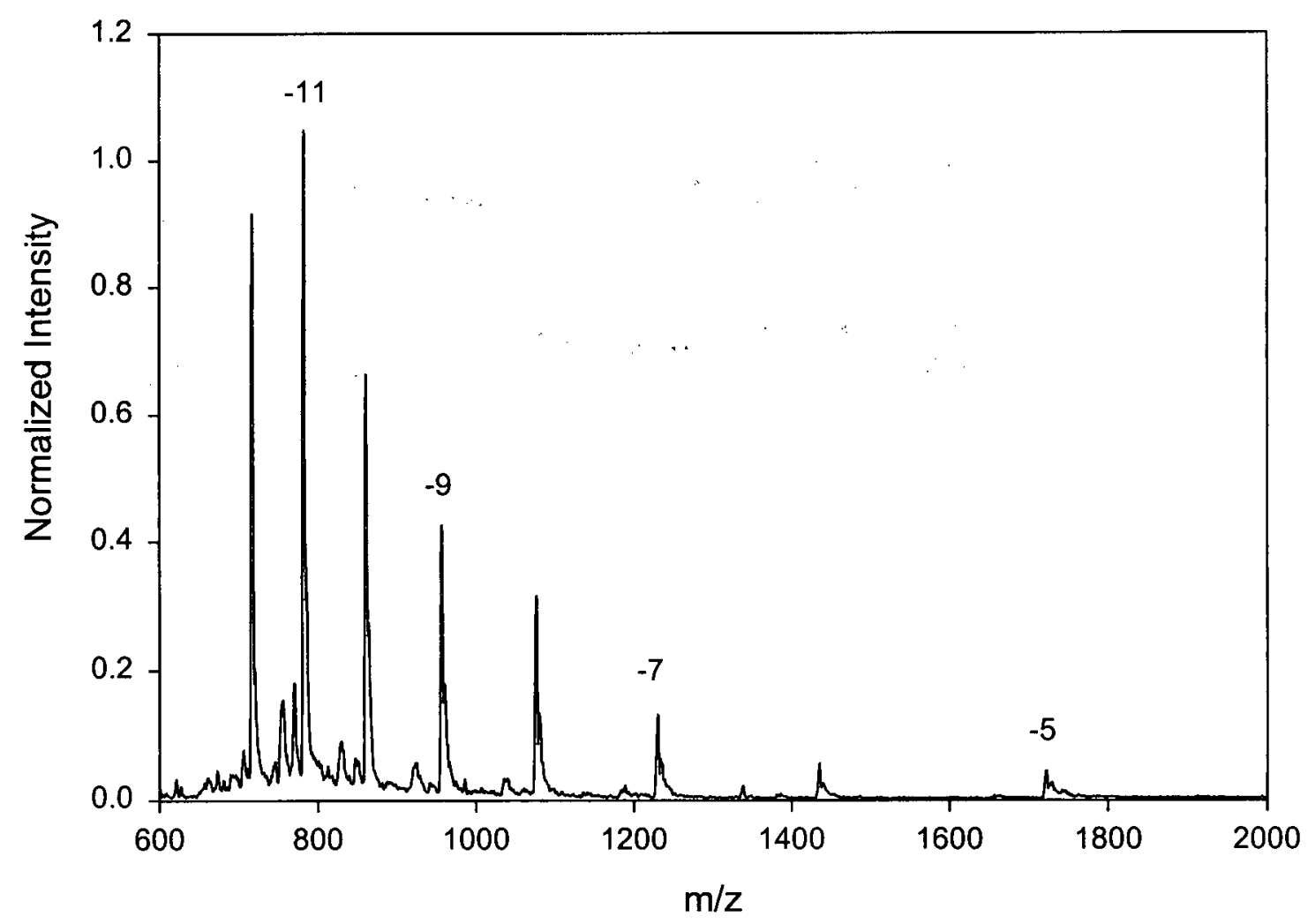

Figure 4-1. Mass spectrum of the 28-mer oligodeoxynucleotide. 


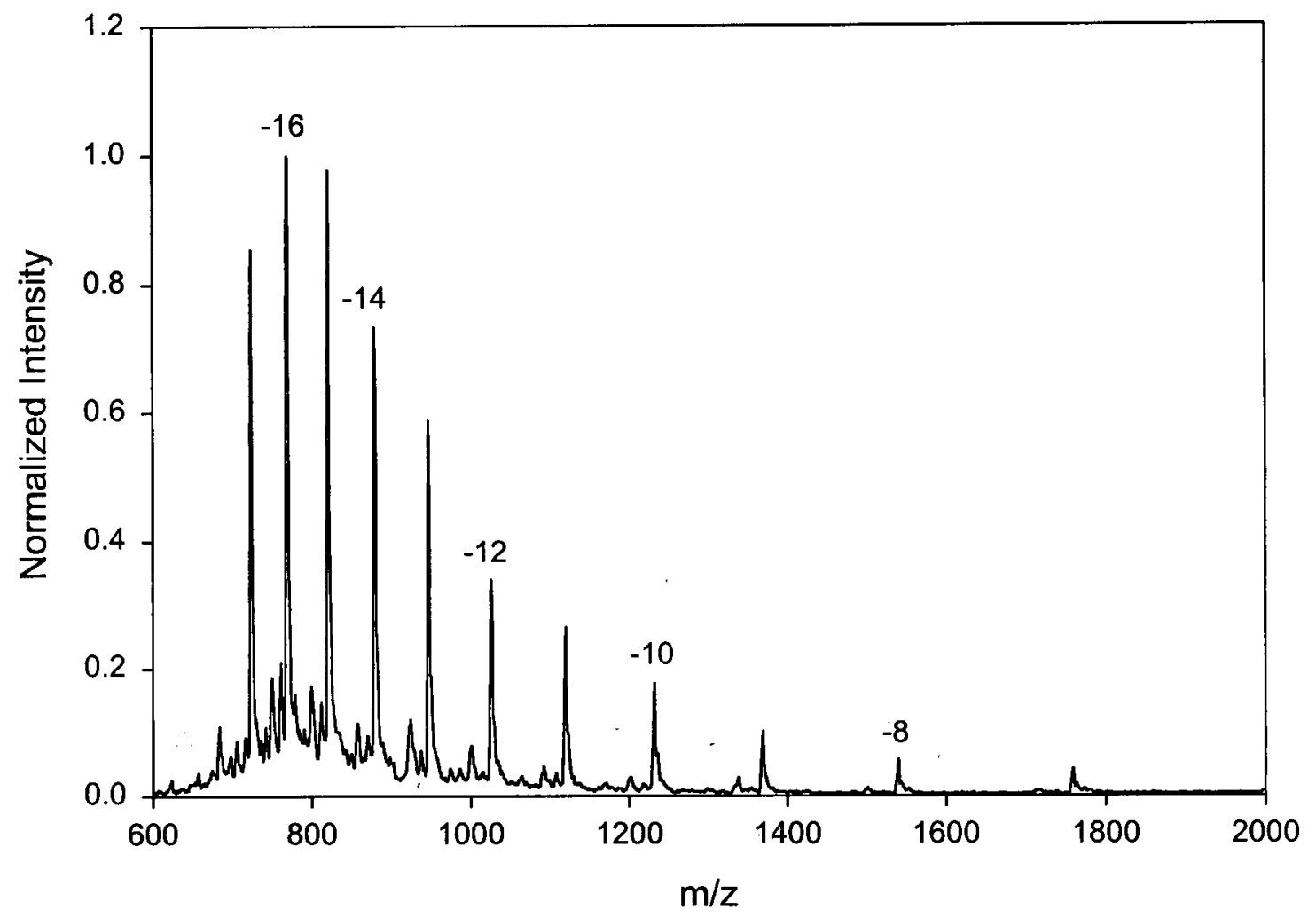

Figure 4-2. Mass spectrum of the 40-mer oligodeoxynucleotide. 


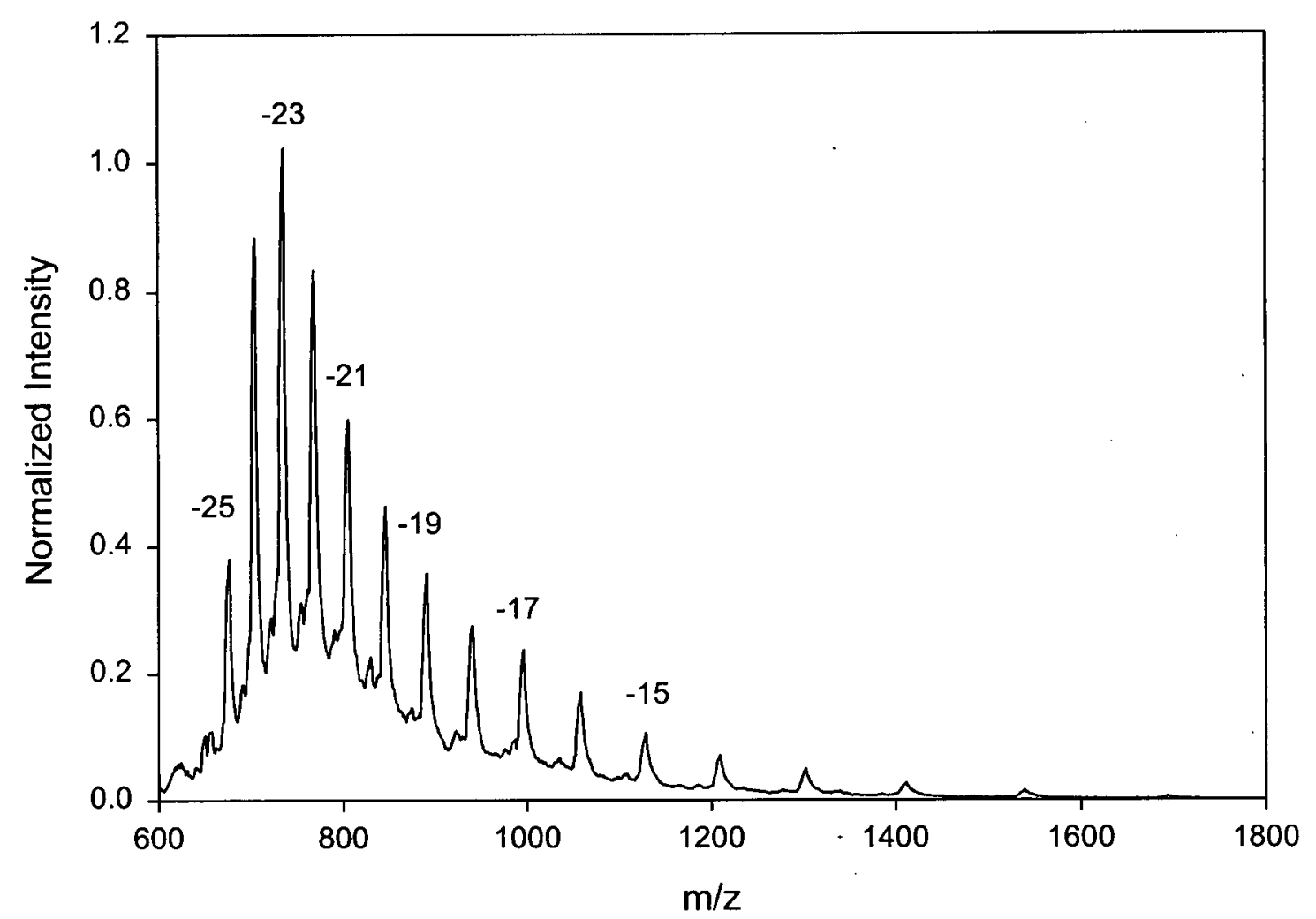

Figure 4-3. Mass spectrum of the 55-mer oligodeoxynucleotide.

\subsubsection{Sensitivity Comparisons of the 28-, 40- and 55-mer Oligodeoxynucleotides}

DNA sequencing today is performed almost exclusively by the enzymatic dideoxyribonucleotide termination (Sanger) concept, where the resulting sequencing products are separated on a polyacrylamide gel. The gel casting/electrophoresis is the most time consuming and labor intensive step in DNA sequencing and if replaced by MALDI-MS the time could ideally be reduced from several hours to a few minutes [107]. The potential of ESI-MS to verify the synthesis of modified oligonucleotides and analyze PCR products has been demonstrated and very frequently only the mass is needed to confirm the identity of a particular compound 
due to the high mass accuracy obtained with ESI-MS $[108,109]$. The use of ESI with a Fourier transform ion cyclotron resonance mass spectrometer (FTICR), which has a superior mass resolution, enabled the identification and characterization of oligonucleotides up to 120 bases [110]. However there are some limitations to both methods. Both ESI and MALDI mass spectra of oligonucleotides strongly suffer from metal cation adduction and, therefore, replacement of metal cations by ammonium ions or other methods that are mentioned in chapter 1 are critical in the sample preparation.

The production of a variety of charge states in ESI compromises the analysis of complex mixtures of oligonucleotides [36]. For MALDI, ion fragmentation is the most pronounced problem. A high tendency of oligonucleotide ions to fragment, strongly increasing with increasing molecular weight has been observed in several studies $[111,112,113]$. This general loss of signal with increasing size has been also seen in the ESI analysis of oligonucleotides [68]. A number of possible explanations exist for this behavior including the following: a similar instrumental and/or detector bias toward smaller oligonucleotides; increased cation adduction of larger oligonucleotides, resulting in a dividing of their signal over many peaks; differences in the collisional cross sections between larger and smaller oligonucleotides. If larger oligonucleotides had substantially larger cross sections than smaller oligonucleotides, they would have more collisions and could have greater losses in high-pressure regions of a mass spectrometer. However, in this study, the of 28-, 40- and 55-mer oligodexoynucleotides ESI spectra show similar sensitivities and a fall-off in signal intensity with increasing mass has not been observed. The sensitivities of the spectra in Figures 4-1, 4-2 and 4-3 corresponding to 28-, 40- and 55-mer oligodeoxynucleotides are $1.4 \times 10^{6}$ ions $/ \mathrm{s}, 1.3 \times 10^{6}$ ion $/ \mathrm{s}$ and $1.12 \times 10^{6}$ ions $/ \mathrm{s}$, respectively. 


\subsection{Mass Spectra of Ubiquitin, Cytochrome $c$ and Myoglobin}

Ubiqutin, cytochrome $c$ and myoglobin were chosen for study because they have similar molecular weights to the oligodeoxynucleotides and do not contain disulphide bonds. Ubiqutin, cytochrome $c$ and myoglobin are globular proteins. Ubiqutin contains 76, cytochrome $c 104$ and myoglobin 153 amino acid residues. Cytochrome $c$ and myoglobin contain a heme group. In cytochrome $c$ heme group is bounded covalentley and in myoglobin non-covalently. The proteins were denatured in solution and so produced relatively high charge states [20]. When myoglobin is denatured by acid or organic solvent in solution, the heme group is removed from the protein. As a result, only apomyoglobin (M.W. 16951) ions were observed in mass spectrum. For cytochrome $\mathrm{c}$, addition of acid and organic solvent can cause denaturation of the protein, but the heme group remains bonded to the protein. ESI-mass spectra of ubiqutin, cytochrome $c$ and myoglobin at an orifice-skimmer voltage difference of $110 \mathrm{~V}$ are shown in Figure 4-4, 4-5 and 4-6, respectively. For ubiquitin (M.W. 8,565) eight charge states, $\{+5\}$ to $\{+12\}$ were produced corresponding to $\mathrm{m} / \mathrm{z}: 1713\{+5\}, 1428\{+6\}, 1224\{+7\}, 1070\{+8], 952$ $\{+9\}, 857\{+10\}, 779\{+11\}, 715\{+12\}$. For cytochrome $c$ (M.W. 12,380) eleven charge states, $\{+8\}$ to $\{+18\}$ were produced corresponding to $\mathrm{m} / \mathrm{z}: 1545\{+8\}, 1374\{+9\}, 1236\{+10\}, 1124$ $\{+11\}, 1031\{+12\}, 951\{+13\}, 883\{+14\}, 825\{+15\}, 773\{+16\}, 728\{+17\}, 687\{+18\}$. For myoglobin (M.W. 17,568) thirteen charge states, $\{+13\}$ to $\{+25\}$ were produced corresponding to $\mathrm{m} / \mathrm{z}: 1304\{+13\}, 1211\{+14\}, 1130\{+15\}, 1060\{+16\}, 997\{+17\}, 942\{+18\}, 893\{+19\}$, $848\{+20\}, 808\{+21\}, 771\{+22\}, 737\{+23\}, 707\{+24\}, 679\{+25\}$. 


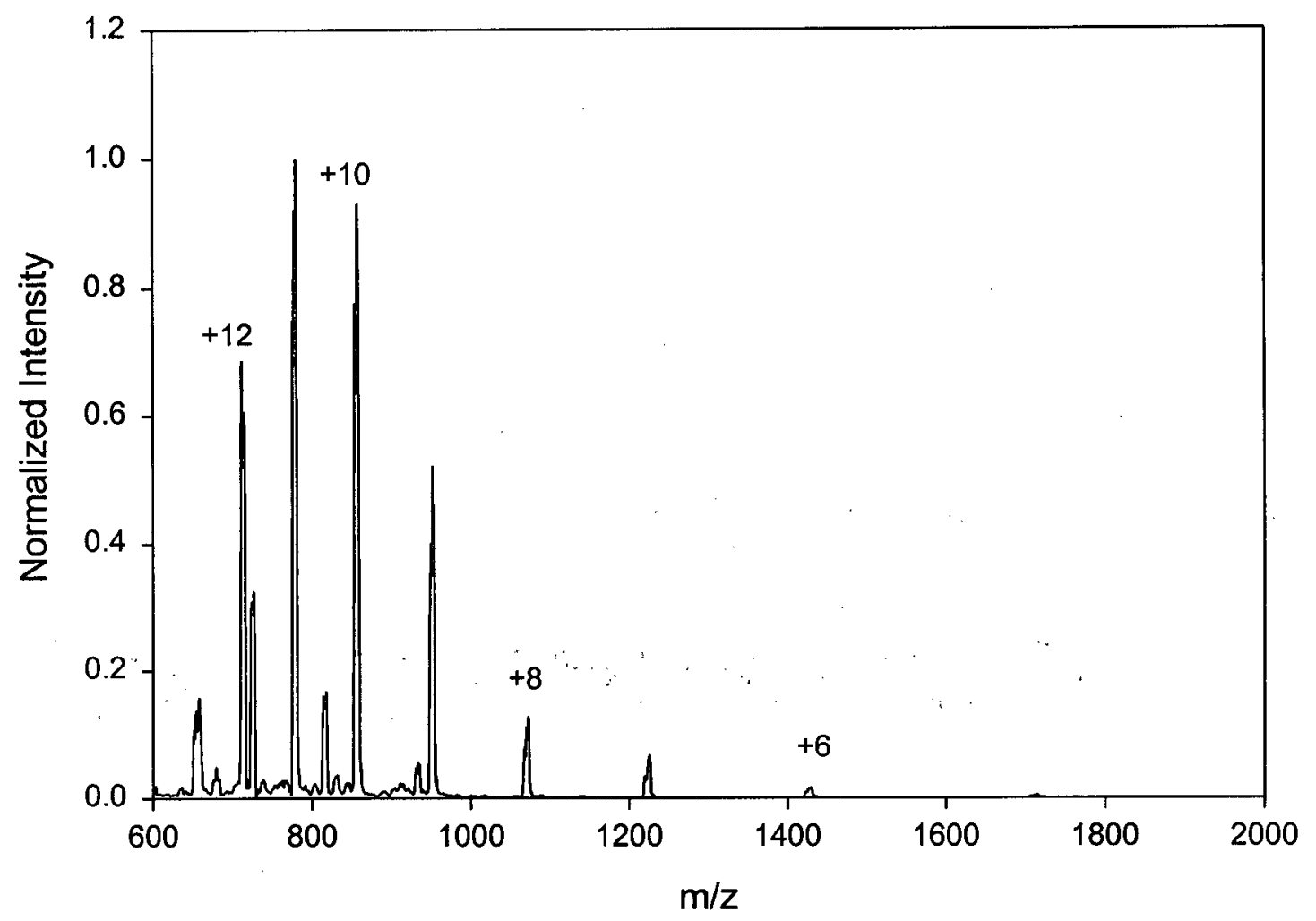

Figure 4-4. Mass spectrum of ubiqutin.

As can be noticed from the mass spectra of the DNA and protein samples, the DNA samples produced negative charge states in ESI that are similar to those produced in positive mode by denatured proteins of the same molecular weight. The average charges on ubiquitin, cytochrome $\mathrm{c}$ and apomyoglobin are $+10.5,+14.6$ and +18.0 and the average charge on the $28-$, 40 - and $55-m e r$ are $-10.3,-14.3$ and -21.3 , respectively. The average charge is calculated as: $\sum_{i} N_{i} I_{i} / \sum_{i} I_{i}$

Where $\mathrm{N}_{\mathrm{i}}$ is the number of charges on a peak in the spectrum and $\mathrm{I}_{\mathrm{i}}$ is the relative intensity of the peak. With proteins protonation is limited primarily to the $\mathrm{N}$-terminus and basic side chains. With oligodeoxynucleotides, each nucleotide contains a phosphate group and can in principle 
contribute one negative charge. For the DNA samples, the numbers of charges per nucleotide range from about 0.2 to 0.5 with an average of 0.37 , an average of one charge for every 2.7 nucleotides. For the proteins there is one charge for about every 7.5 residues.

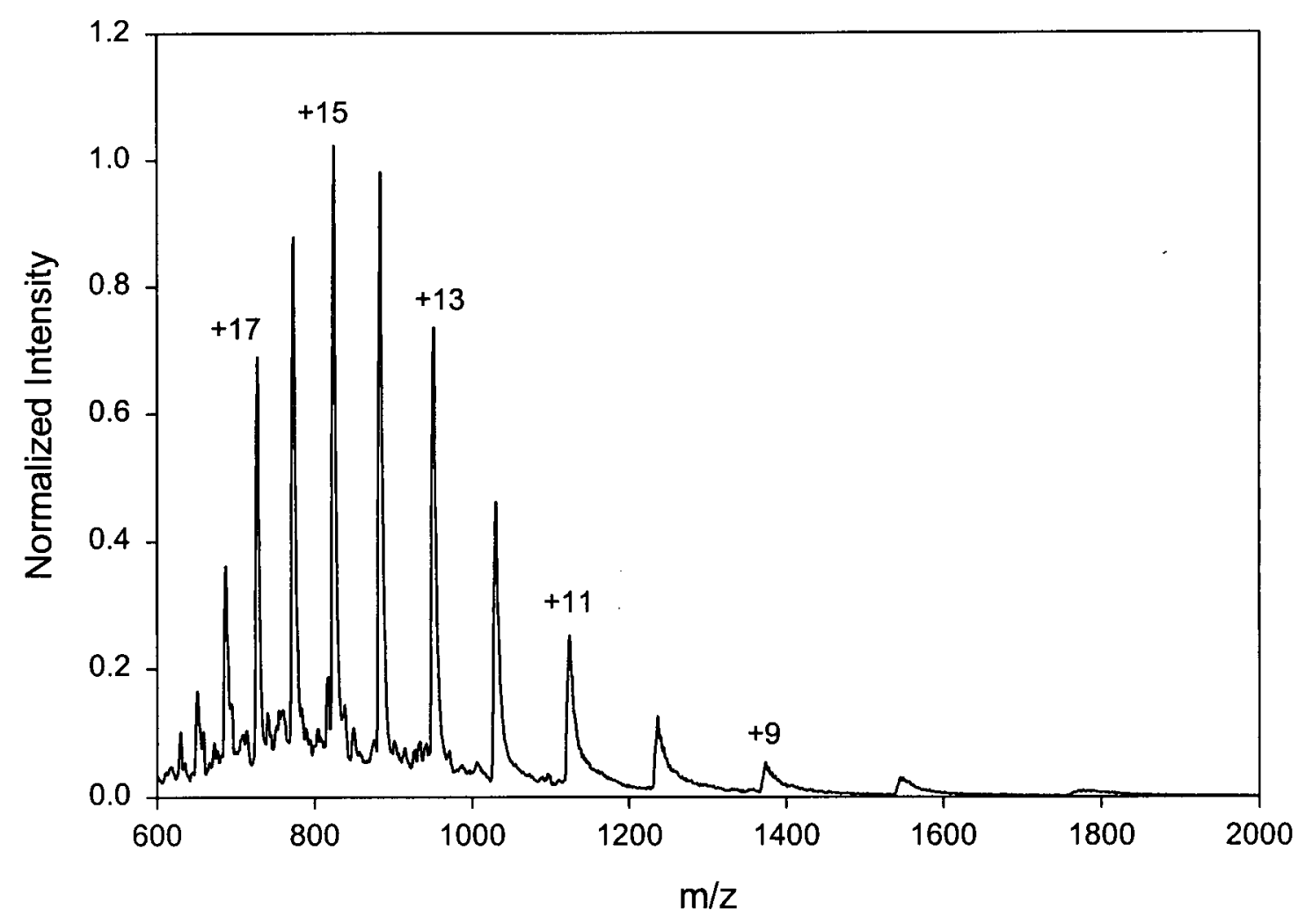

Figure 4-5. Mass spectrum of cytochrome $c$. 


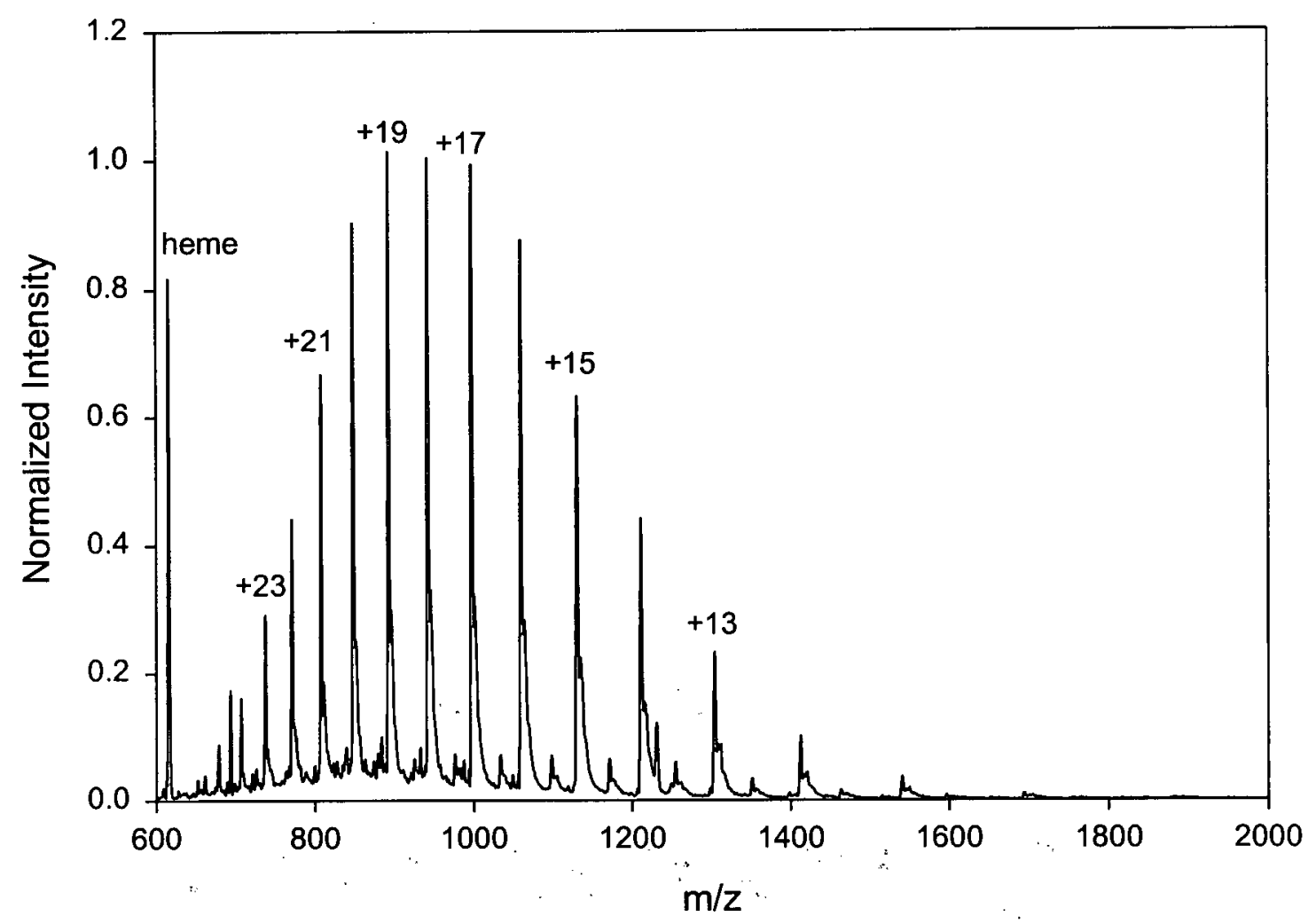

Figure 4-6. Mass spectrum of myoglobin.

\subsection{DNA and Protein Ion Cross Sections}

Collision cross sections of ions of the 28-mer and ubiquitin are shown in Figure 4-7, of ions of the 40-mer and cytochrome $c$ in Figure 4-8 and of ions of the 55-mer and apomyoglobin in Figure 4-9. The DNA cross sections in these Figures are the average of three measurements over several months and the error bars are the relative standard deviations. Collision cross sections for the proteins were measured once and so statistical uncertainties are not shown. However, the cytochrome $\mathrm{c}$ and apomyoglobin cross sections measured here agree within a few percent with those measured here previously by the same method [64]. The drag coefficients 
used to calculate the DNA and protein cross sections are calculated using (equation 2.14) are in Table 4-1 and 4-2 respectively.

Table 4-1 Drag Coefficients of 28-, 40- and 55-mer Oligodeoxynucleotide Ions

\begin{tabular}{|l|l|l|l|l|l|}
\hline Charge State & $\mathbf{C}_{\mathbf{D}}$ 28- mer & Charge State & $\mathbf{C}_{\mathbf{D}}$ 44- mer & Charge State & $\mathbf{C}_{\mathbf{D}}$ 55- mer \\
\hline-5 & 2.604 & -7 & 2.613 & -15 & 2.458 \\
\hline-6 & 2.536 & -8 & 2.561 & -16 & 2.439 \\
\hline-7 & 2.484 & -9 & 2.519 & -17 & 2.422 \\
\hline-8 & 2.444 & -10 & 2.485 & -18 & 2.407 \\
\hline-9 & 2.412 & -11 & 2.455 & -19 & 2.393 \\
\hline-10 & 2.385 & -12 & 2.430 & -20 & 2.380 \\
\hline-11 & 2.362 & -13 & 2.409 & -21 & 2.369 \\
\hline-12 & 2.343 & -14 & 2.390 & -22 & 2.358 \\
\hline & & -15 & 2.373 & -23 & 2.348 \\
\hline & & -16 & 2.358 & -24 & 2.339 \\
\hline & & -17 & 2.345 & -25 & 2.322 \\
\hline
\end{tabular}


Table 4-2 Drag Coefficients of Ubiquitin, Cytochrome $c$ and Myoglobin Ions

\begin{tabular}{|l|l|l|l|l|l|}
\hline Charge State & Ubiquitin & Charge State & Cytochrome c & Charge State & Myoglobin \\
\hline+5 & 2.602 & +9 & 2.524 & +13 & 2.503 \\
\hline+6 & 2.534 & +10 & 2.489 & +14 & 2.479 \\
\hline+7 & 2.483 & +11 & 2.459 & +15 & 2.458 \\
\hline+8 & 2.442 & +12 & 2.434 & +16 & 2.440 \\
\hline+9 & 2.410 & +13 & 2.412 & +17 & 2.423 \\
\hline+10 & 2.384 & +14 & 2.393 & +18 & 2.408 \\
\hline+11 & 2.361 & +15 & 2.376 & +19 & 2.394 \\
\hline+12 & 2.342 & +16 & 2.361 & +20 & 2.381 \\
\hline & & +17 & 2.348 & +21 & 2.370 \\
\hline & & & & +22 & 2.359 \\
\hline & & & & +23 & 2.349 \\
\hline & & & & & +24 \\
\hline
\end{tabular}




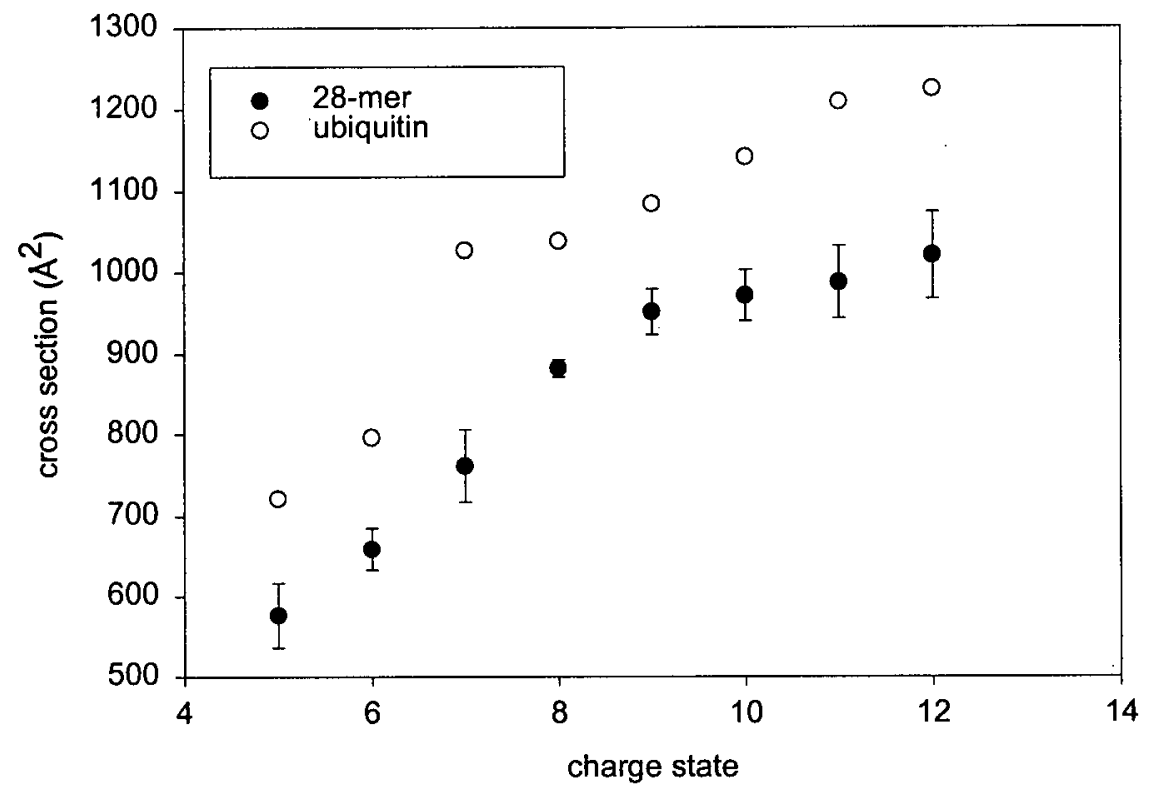

Figure 4-7. Collision cross section vs. charge state of ions of ubiquitin and 28-mer.

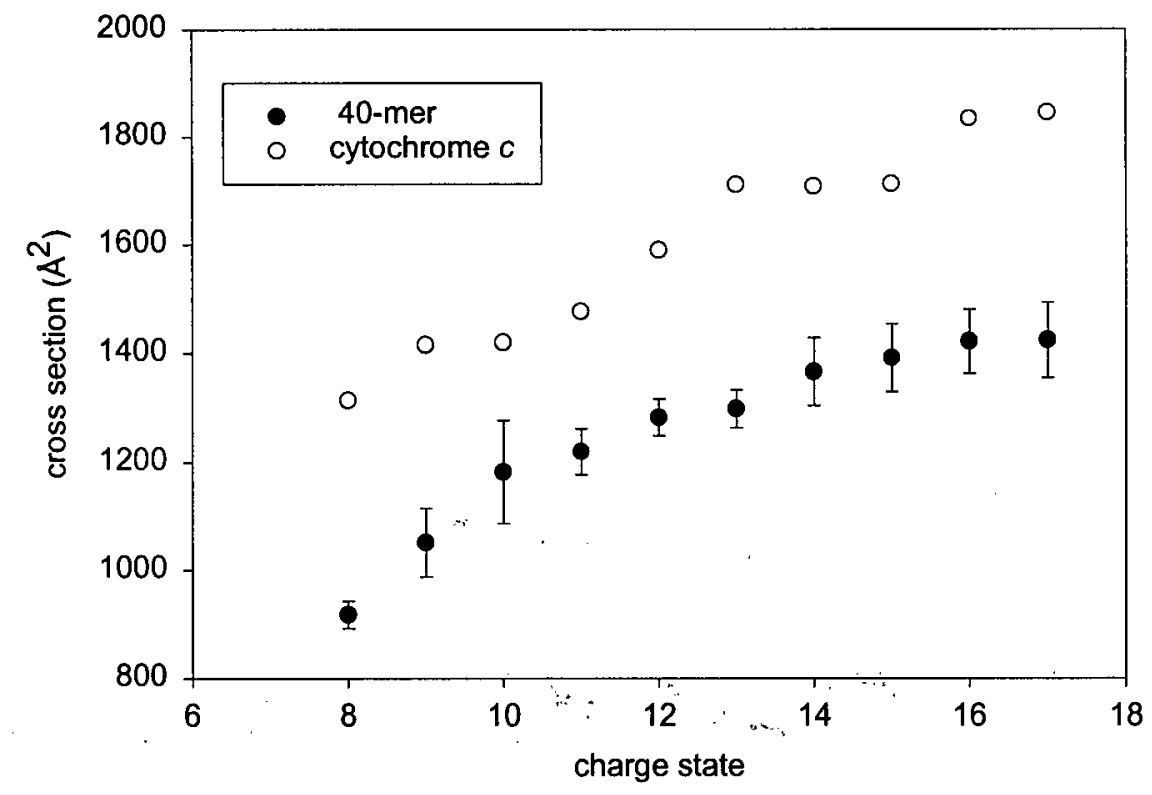

Figure 4-8. Collision cross section vs. charge state of ions of cytochrome $c$ and 40-mer. 


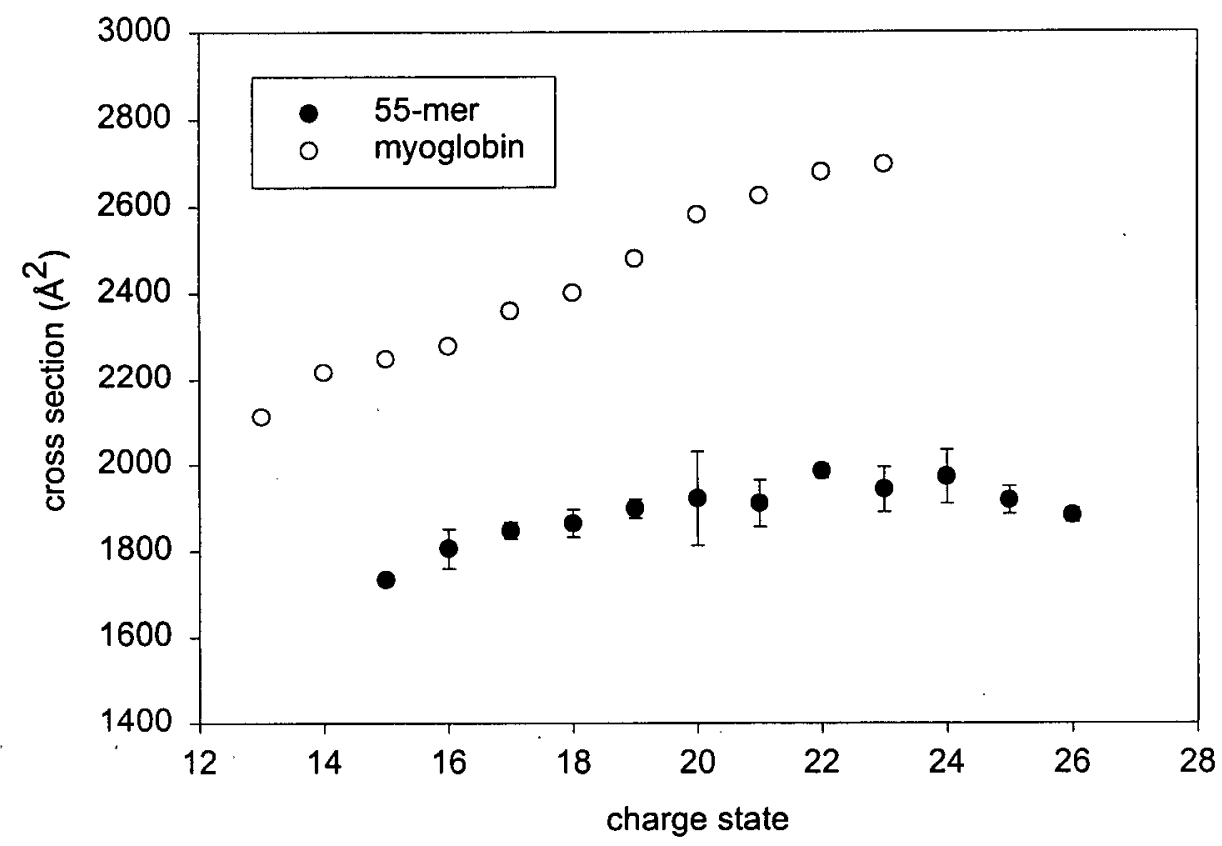

Figure 4-9. Collision cross section vs. charge state of ions of apomyoglobin and 55-mer.

Ion mobility experiments have been used to determine cross sections for ubiquitin [93], cytochrome $c$ [114] and apomyoglobin [91]. In each case, the reported cross sections were determined from the mobility model, in which collisions were treated as hard sphere collisions. It was argued in chapter 2 that a more realistic model involves diffuse scattering. Because the average drag force on an ion with diffuse scattering is greater by a factor of 1.35 , to obtain the projection area with diffuse scattering the hard sphere cross sections should be reduced by a factor of 0.74 . This produces results similar to the EHSS model, which requires that cross sections determined by the hard spheres equation be reduced by more than $20 \%$ [ 91$]$. When the hard sphere cross sections of $[22,23,115]$ are reduced by a factor of 0.74 , the cross sections for ubiquitin in Figure 4-7 are found to be $0.96 \pm 0.07$ on average of those determined by mobility 
[93]. Averaged over charge states, the cross sections of cytochrome $c$ in Figure 4-8 are $0.91 \pm 0.04$ of those determined by mobility [114] and the cross sections of apomyoglobin in Figure $4-9$ are $0.94 \pm 0.01$ of those determined by mobility [ 91 ]. For the charge states $+5,+6,+7$, +8 of ubiqutin and $+8,+9$ of cytochrome $c$, where multiple conformations were detected by mobility, the cross sections here are closest to those of the most compact conformers. The small differences between the energy loss and mobility experiments could easily be attributed to differences in the scattering models used or to the differences in the collision dynamics at the low and intermediate energies used in ion mobility and energy loss experiments, respectively. If for example, a factor of 0.69 is used to correct the hard sphere cross sections as suggested by Epstein's scattering model [81] the energy loss cross sections and mobility cross sections agree within a few percent. The dynamics of scattering of protein ions by Ar or He is not fully understood, and this introduces an uncertainty in converting energy loss or mobility measurements to projection areas or collision cross sections $[64,91,116,117]$.

In Figures 4-7 , 4-8 and 4-9, it is seen that oligodeoxynucleotide ions of a given charge and molecular weight have significantly smaller collision cross sections than positive ions of proteins of the same molecular weight. The difference cannot be attributed to the difference in ion polarity. Negative ions of these proteins have similar cross sections to positive ions of the same charge state [57]. Averaged over charge states, the cross sections of the 28-mer are $0.824 \pm 0.041$ of the ubiquitin cross sections, the cross sections of the 40 -mer are $0.782 \pm 0.041$ of the cross sections of the cytochrome $\mathrm{c}$ and the cross sections of the 55 -mer are $0.758 \pm 0.025$ of the cross sections of apomyoglobin. There is a slight but significant decrease in the DNA cross sections relative to the proteins as the molecular weights increase. The cross section of the 
crystal structure of myoglobin has been estimated [91] as $1768 \mathrm{~A}^{2}$, a value close to those measured here for both the protein and 55-mer ions.

In principle, the difference between the protein and DNA cross sections could be caused by differences in the collision dynamics of these species. The diffuse scattering model used to calculate cross sections corresponds to highly inelastic collisions. The evidence that proteinargon collisions are highly inelastic at collision energies near those used in the energy loss experiments is reviewed in [63]. If collisions of ions of oligodeoxynucleotides with Ar were completely elastic (hard spheres) the drag coefficient should be calculated for specular or elastic collisions. For the DNA ions this would decrease the value of $C_{D}$ by factors between $18 \%$ for the low charge states to $12 \%$ for the high charge states, so that cross sections calculated from equation (2.20) would increase by the same amount. This would reduce but not eliminate the differences in cross sections between proteins and DNA ions. There is, however, no reason to believe that oligodeoxynucleotide ions would have completely elastic collisions while protein ions had highly inelastic collisions at the same collision energies.

Shelimov and Jarrold, in a study of apomyoglobin ions, proposed that if high charge state ions from different proteins have string-like structures, the cross section per residue should be a universal function of the number of charges per residue [91]. They noted that high charge states of cytochrome $\mathrm{c}$ and apomyoglobin showed this behavior, even though the cross sections were significantly smaller than those calculated for the fully stretched out proteins. Conservely, if the ions had globular or approximately spherical structures a plot of cross section / (number of residues $)^{2 / 3}$ should be a universal function of charge / (number of residues) $)^{2 / 3}$. Low charge states of cytochrome $\mathrm{c}$ and apomyoglobin showed this behavior. Plots showing both the cross section 
per nucleotide vs. charge per nucleotide and cross section per (nucleotide) ${ }^{2 / 3}$ vs. charge per (nucleotide) $^{2 / 3}$ for the DNA ions studied here are shown in Figure 4-10, and 4-11, respectively.

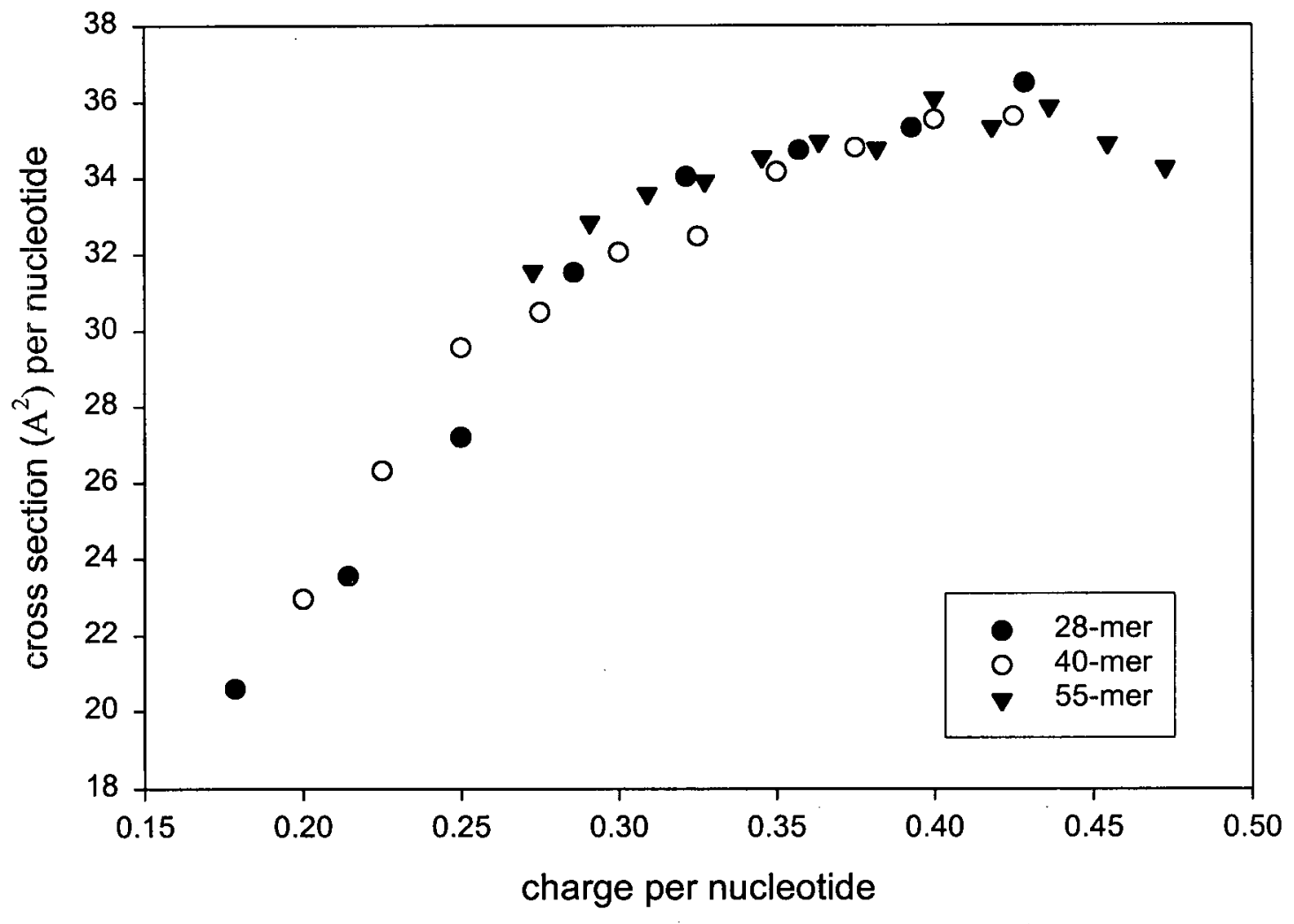

Figure 4-10. Cross section per nucleotide vs. charge per nucleotide for ions of the 28-, 40- and 55 -mer. 


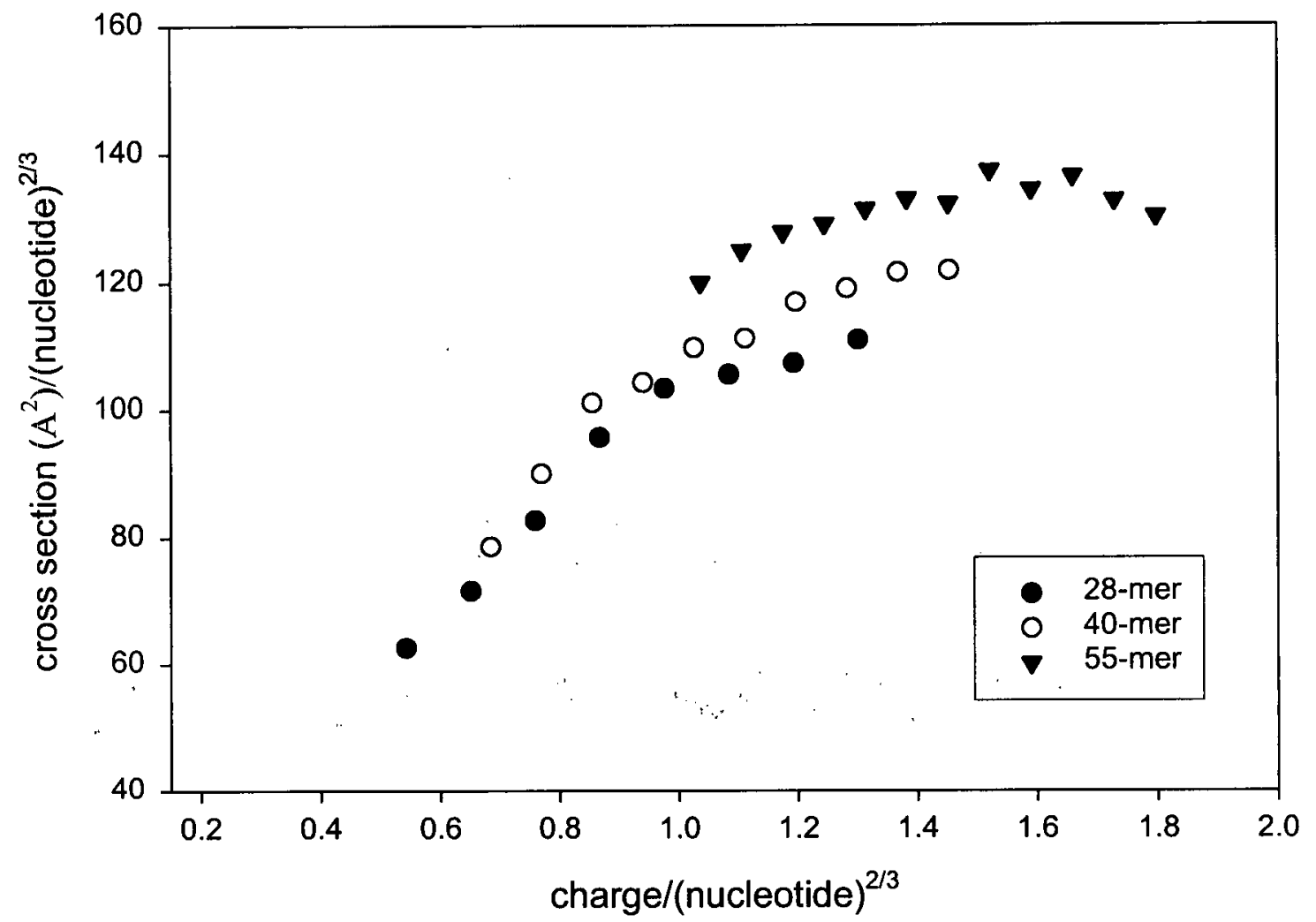

Figure 4-11. Cross section / (number of nucleotides) $)^{2 / 3}$ vs. charge / (number of nucleotides) ${ }^{2 / 3}$ for ions of the 28-, 40- and 55-mer.

It is seen that the higher charge state ions from oligodeoxynucleotides of varying lengths exhibit a smaller deviation from one another in the plots which are normalized per nucleotide, Figure 4-10, than in the plots which are normalized per (nucleotide) ${ }^{2 / 3}$, Figure $4-11$. The opposite is true for the lower charge states. Based on the work of Shelimov and Jarrold, this implies that the lower charge states have a globular or spherical conformations while the higher charge states have a non-globular or extended conformation.

As mentioned earlier a main driving force for this work was to see if the fall-off in signal intensity with increasing mass as observed in some MALDI and ESI experiments was due to 
differences in the transfer efficiency of protein and DNA ions from the ionization region to the mass analyzer. Such differences if they exist, might stem from differences in collision cross sections. The results in Figures 4-7,4-8 and 4-9 show that nucleic acids behave similarly to proteins. The relatively small differences in cross sections seen here are unlikely to explain the losses of ion signal for high mass polynucleotide ions. 


\section{Chapter 5 Future Work}

Cross sections of oligonucleotides provide insight into the gas-phase conformations of these ions. However, determining the structures of these ions in gas-phase is a greater challenge, because the solution structures are often less well understood. Secondary structure prediction for any given sequence relies on energy minimization algorithms. Due to the constraints of current mathematical models and the uncertainties in the thermodynamic parameters employed, the ability to accurately predict polynucleotide secondary structure is limited, making comparisons of solution and gas phase behavior more difficult [118]. However, single stranded polynucleotides that are self-complementary, studied with additional methods such as H/D exchange, may allow further insights into the gas phase ion structures in future studies. 


\section{References}

1. J.J. Thomson, Rays of Positive Electricity and Their Application to Chemical Analysis, Longmans, Green \& Co., London, 1913.

2. Paul, W., Steinwedel, H. Z., Naturforsch., 1953, 8a, 448.

3. Fenn, J.B., Mann, M., Meng, C.K., Wong, S.F., Whitehouse, C.M., Science, 1989, 246, 64.

4. Karas, M., Hillenkamp, F., Anal. Chem., 1988, 60, 2299.

5. Dole, M., Mack, L.L., Hines, R.L., Mobley, R.C., Ferguson, L.D., Alice, M.B., J. Chem. Phys., 1968, 49, 2240.

6. Schmelzeisen-Redeker, G., Giesmann, U., Röllgen, F.W., J. Phys. Colloq.,1984, 45, Supplement 12, C9-297.

7. Iribarne, J.V., Thomson, B.A., J. Chem. Phys., 1976, 64, 2287.

8. Cole, R.B. Ed. Electrospray Ionization Mass Spectrometry, John Wiley \& Sons, Inc.: New York, 1997.

9. Yamashita, M., Fenn, J.B., J. Phys. Chem., 1984, 88, 4451.

10. Covey, T.R., Bonner, R.F., Sushan, B.I., Henion, J.D., Rapid Commun. Mass Spectrom., 1988, 2, 249.

11. Fenn, J.B., Mann, M., Meng, C.K., Wong, S.F., Whitehouse, C.M., Mass Spectrom. Rev., 1990, 9, 37.

12. Bruins, A.P., Covey, T.R., Henion, J.D., Anal. Chem., 1987, 59, 2642.

13. Olivares, J.A., Nguyen, N.T., Yonker, C.R., Smith, R.D., Anal. Chem., 1987, 59, 1230.

14. Wilm, M., Mann, M., Anal. Chem., 1996, 68, 1. 
15. Yamashita, M., Fenn, J.B., J. Phys. Chem., 1984, 88, 4671.

16. Chowdhury, S.K., Katta, V., Chait, B.T., Rapid Commun. Mass Spectrom., 1990, 4, 81.

17. Douglas, D.J., French, J.B., J. Am. Soc. Mass Spectrom., 1992, 3, 398.

18. Mann, M., Meng, C.K., Fenn, J.B., Anal. Chem., 1989, 61, 1702.

19. Creighton, T.E., Proteins: Structure and Molecular Principles, W.H. Freeman: New York, 1993.

20. Chowdhury, S.K., Katta, V., Chait, B.T.,.J. Am. Chem. Soc., 1990, 112, 9012.

21. Mirza, U.A., Chait, B.T., Int. J. Mass Spectrom. Ion Processes, 1997, 162, 173.

22. Konerman, L., Douglas, D.J., Biochemistry, 1997, 36, 12296.

23. Feng, R., Konishi, Y., J. Am. Soc. Mass Spectrom., 1993, 4, 638.

24. Loo, J.A., Ogorzalek Loo, R.R., Udseth, H.R., Edmonds, C.G., Smith, R.D., Rapid Commun. Mass. Spetrom., 1991, 5, 101

25. Babu, K.R., Moradian, A., Douglas, D.J., J. Am. Soc. Mass Spectrom., 2001, $12,317$.

26. Loo, J.A., Edmonds, C.G., Udseth, H.R., Smith, R.D., Anal. Chem., 1990, 62, 693.

27. Konerman, L., Collings, B.A., Douglas, D.J., Biochemistry, 1997, 36, 5554.

28. Stryer,L., Biochemistry, W.H. Freeman and Company: San Francisco, 1981.

29. Smith, R.D., Loo, J.A., Edmonds, C.G., Barinaga, C.J., Udseth, H.R., Anal. Chem., $1990,62,882$.

30. Stults, J.T., Marsters, J.C., Rapid Commun. Mass Spectrom., 1991, 5, 359.

31. Limbach, P.A., Crain, P.F., McCloskey, J.A., J. Am. Soc. Mass Spectrom., 1995, 6, 27.

32. Greig, M., Griffey, R.H., Rapid Commun. Mass Spectrom., 1995, 9, 97.

33. Tong, X., Henion, J., Ganem, B., J. Mass Spectrom., 1995, 30, 867.

34. Griffey, R.H., Sasmor, H., Greig, M.J., J. Am. Soc. Mass Spetrom., 1997, 8, 155. 
35. Apffel, A., Chakel, J.A., Fischer, S., Lichtenwalter, K., Hancock, W.S., Anal. Chem., $1997,69,1320$.

36. Wunschel, D.S., Fox, K.F., Fox, A., Bruce, J.E., Muddiman, D.C., Smith, R.D., Rapid Commun. Mass Spectrom., 1996, 10, 29.

37. Harrison, A.G., Mass Spectrom. Rev., 1997, 16, 201.

38. Green, M.K., Lebrilla, C.B., Mass Spectrom. Rev., 1997, 16, 53.

39. Gross, D.S., Williams, E.R., J. Am. Chem. Soc., 1995, 117, 883.

40. Gross, D.S., Schnier, P.D., Rodriguez-Cruz, S.E., Fagerquist, C.K., Williams, E.R., Proc. Nat. Acad. Sci. USA, 1996, 93, 3143.

41. Schnier, P.D., Gross, D.S., Williams, E.R., J. Am. Soc. Mass Spectrom., 1995, 6, 1086.

42. McLucky, S.A., Van Berkel, G.J., Glish, G.L., J. Am. Chem. Soc., 1990, 112, 5668.

43. Ogorzalek Loo, R.R., Smith, R.D., J. Am. Soc. Mass Spectrom., 1994, 5, 207.

44. Ogorzalek Loo, R.R., Loo, J.A., Udseth, H.R., Fulton, J.L., Smith, R.D., Rapid Commun. Mass Spectrom., 1992, 6, 159.

45. Herron, W.J., Goeringer, D.E., McLucky, S.A., J. Am. Soc. Mass Spectrom., 1995, 6, 529.

46. Smith, D.L., Deng, Y., Zhang, Z., J. Mass Spectrom., 1997, 32,135.

47. Chait, B.T., Katta, V., Rapid Commun. Mass Spectrom., 1991, 5, 214.

48. Miranker, A., Robinson, C.V., Radford, S.E., Aplin, R.T., Dobson, C.M., Science, 1993, 262, 896.

49. Robinson, C.V..GroB, M., Eyles, S.J., Ewbank, J.J., Mayhew, M., Hartl, F.U., Dobson, C.M., Radford, S.E., Nature, 1994, 372, 646. 
50. Winger, B.E., Light-Wahl, K.J., Rockwood, A.L., Smith, R.D., J. Am. Chem. Soc., 1992, 114, 5897.

51. Suckau, D., Shi, Y., Beu, S.C., Senko, M.W., Quinn, J.P., Wampler III, F.M., McLafferty, F.W., Proc. Nat. Acad. Sci. USA, 1993, 90, 790.

52. Wood, T.D., Chorush, R.A., Wampler III, F.M., Little, D.P., O’Conner, P.B., .McLafferty, F.W., Proc. Nat. Acad. Sci. USA, 1995, 92, 2451.

53. Hemling, M.E., Conboy, J.J., Bean, M.F., Mentzer, M., Carr, S.A., J. Am. Soc. Mass Spectrom., 1994, 5, 434.

54. Robinson, J.M., Greig, M.J., Griffey, R.H., Mohan, V., Laude, D.A., Anal. Chem., 1998, 70, 3566.

55. Freitas, M., Shi, S.D.-H, Hendrickson, C.L., Marshall, A.G., J. Am. Chem. Soc., 1998, $120,19187$.

56. Hofstadler, S.A., Sannes-Lowery, K.A., Griffey, R.H., J. Mass Spectrom., 2000, 35, 62.

57. Hoaglund-Hyzer, C.S., Counterman, A.E., Clemmer, D.E., Chem. Rev., 1999, 99, 3037.

58. Chernusevich, I.V., Verentchikov, A.N., Ens, W., Standing, K.G., J. Am. Soc. Mass Spectrom., 1996, 7, 342.

59. Thomson, B.A., Douglas, D.J., Corr, J.J., Hager, J.W., Jolliffe, C.L., Anal. Chem., 1995, 34, 1626.

60. Clemmer, D.E., Jarrold, M.F., J. Mass Spectrom., 1997, 32, 577.

61. Covey, T., Douglas, D.J., J. Am. Soc. Mass. Spectrom., 1993, 4, 616. 
62. Reimann, C.T., Sullivan, P.A., Axelsson, J., Quist, A.P., Altman, S., Roepstorff, P., Velazquez, I., Tapia, O., J. Am. Chem. Soc., 1998, 120, 7608.

63. Chen, Y.-L., Mauk, M.R., Mauk, A.G., Douglas, D.J., J. Am. Soc. Mass. Spectrom., $2002,13,59$.

64. Chen, Y.-L., Collings, B.A., Douglas, D.J., J. Am. Soc. Mass Spectrom., 1997, 8, 681.

65. Hoaglund, C.S., Liu, Y., Ellington, A.D., Pagel, M., Clemmer, D.E., J. Am. Chem. Soc., 1997, 119, 9051.

66. Fitzgerald, M.C., Zhu, L., Smith, L.M., Rapid Commun. Mass Spectrom., 1993, 7, 895.

67. Smith, L.M., Nature Biotechnol., 1996, 14, 1084.

68. Scalf, M., Westphall, M.S., Smith, L.M., Anal. Chem., 2000, 72, 52.

69. Hayes, R.N., Gross, M.L., Methods in Enzymology, McCloskey J.A., Ed., Vol. 193, 1990, Academic Press: U.S.A

70. Tolmachev, A.V., Chernushevich, I.V., Dodonov, A.F., Standing, K.G., Nucl. Instrum. Methods Phys. Res. B, 1997, 124, 112.

71. Mason, E.A., McDaniel, E.W., Transport Properties of Ions in Gases, Wiley: New York, 1988.

72. Cox, K.A., Julian, R.K., Cooks, R.G., Kaiser, R.E., J. Am. Soc. Mass Spectrom., 1994, $5,127$.

73. Douglas, D.J., J. Am. Soc. Mass Spectrom., 1994, 5, 17.

74. Collings, B.A., Douglas, D.J., J. Am. Chem. Soc., 1996, 118, 4488.

75. Douglas, D.J., J. Phys. Chem., 1982, 86, 185.

76. Halliday, D., Resnick, R., Fundamentals of Physics, Wiley: New York, 1988. 
77. Hersh, A.S., Friichtenicht, J.F., Slattery, J.C., In Rarefied Gas Dynamics, Trilling, L., Wachman, H.Y., Eds., Academic Press: NewYork, 1969, 757.

78. Schaaf, S.A., Chambre, P.L., Fundamentals of Gas Dynamics, Emmons, H.W., Ed., Princton University Press: New Jersey, 1958.

79. Ashkenas, H., In Rarefied Gas Dynamics, Laurmann, J.A., Ed., Academic Press: New York, 1963, 278.

80. Potter, J.L., Miller, J.T., In Rarefied Gas Dynamics, Trilling, L., Wachman, H.Y., Eds., Academic Press: NewYork, 1969, 723.

81. Epstein, P.S., Phys. Rev., 1923, 23, 710.

82. Stadler, J.R., Zurich, V.J., National Advisory Committee for Aeronautics, Technical Note 2423, 1951.

83. Henderson, C.B., AIAA Journal, 1976, 14, 707.

84. Cohen, M.J., Karasek, F.W., J. Chromatogr. Sci., 1970, 8, 330.

85. Hagen, D.F., Anal. Chem., 1979, 51, 870.

86. Jarrold, M.F., J. Phys. Chem., 1995, 99, 11.

87. Radi, P.P., Von Helden, G., Hsu, M.-T., Kemper, P.R., Bowers, M.T., Int. J. Mass Spectrom. Ion Processes, 1991, 109, 49.

88. Wyttenbach, T., Von Helden, G., Bowers, M.T., J. Am. Chem. Soc., 1996, 118, 8355.

89. Clemmer, D.E., Hudgins, R.R., Jarrold, M.F., J. Am. Chem. Soc., 1995, 117, 10141. b) Shelimov, K.B., Jarrold, M.F., J. Am. Chem. Soc., 1996, 118, 10313.

90. Shelimov, K.B., Jarrold, M.F., Clemmer, D.E., Hudgins, R.R., J. Am. Chem. Soc., $1997,119,2240$.

91. Shelimov, K.B., Jarrold, M.F., J. Am. Chem. Soc., 1997, 119, 2987. 
92. Valentine, S.J., Anderson, J.G., Ellington, A.D., Clemmer, D.E., J. Phys. Chem. B, 1997, 101, 3891.

93. Valentine, S.J., Counterman, A.E., Clemmer, D.E., J. Am. Soc. Mass. Spectrom., 1997, $8,954$.

94. Smith, R.D., Barinaga, C.J., Rapid Commun. Mass Spectrom., 1990, 4, 54.

95. Liu, Y., Valentine, S. J., Counterman, A.E., Hougland, C.S., Clemmer, D.E., Anal. Chem., 1997, 67, A728.

96. Jarrold, M.F., Constant, V.A., Phys. Rev. Lett., 1992, 67, 2994.

97. Mack, E., Jr., J. Am. Chem. Soc., 1925, 47, 2468.

98. Shvartsburg, A.A., Jarrold, M.F., Chem. Phys. Lett., 1996, 261, 86.

99. Mesleh, M.F., Hunter, J.M., Shvartsburg, A.A., Schatz, G.C., Jarrold, M.F., J. Phys. Chem., 1996, 100, 16082.

100. Reimann, C.T., Quist, A.P., Kopniczky, J., Sundqvist, B.U.R., Erlandsson, r., Tengvall, P., Nucl. Instrum. Methods Phys. Res. Sect. B, 1994, 88, 29.

101. Sullivan, P.A., Axelsson, J., Altmann, S., Quist, A.P., Sundqvist, B.U.R., Reinmann, C.T., J. Am. Chem. Soc., 1996, 7, 329.

102. Quist, A.P., Ahlbom, J., Reimann, C.T., Sundqvist, B.U.R., Nucl. Instrum. Methods Phys. Res. Sect. B, 1994, 88, 164.

103. Dawson, P.H., Ed., Quadruple Mass Spectrometry and Its Applications; Elsevier: Amsterdam, 1976, reissued by AIP: Woodbury, NY, 1995.

104. March, R.E., Hughes, R.J., Quadrupole Storage Mass Spectrometry; Chemical analysis Vol. 102, Winerfordner, J.D. Ed.; John Wiley \& Sons: New York, 1989.

105. Schneider, B.B., Chen, D.D.Y., Anal. Chem., 2000, 72, 791. 
106. Ono, T., Scalf, M., Smith, L.M., Nucl. Acids Res., 1997, 25, 4581.

107. Smith, L.M., Science, 1993, 262, 530.

108. Potier, N., Van Dorsselaer, A., Cordier, Y., Roch, O., Bischoff, R., Nucleic Acids Res., $1994,22,3895$.

109. Muddiman, D.C., Wunschel, D.S., Liu, C., Pasa-Tolic, L., Fox, K.F., Anderson, G.A., Smith, R.D., Anal. Chem., 1996, 68, 3705.

110. Aaserud, D.J., Kelleher, N.L., Little, D.P., McLafferty, F.W., J. Am. Soc. Mass Spectrom., 1996, 7, 1266.

111. Zhu, L., Parr, G.R., Fitzgerald, M.C., Nelson, C.M., Smith, L.M., J. Am. Chem. Soc., $1995,117,6048$

112. Mouradian, S., Rank, D.R., Smith, L.M., Rapid Commun. Mass Spectrom., 1996, 10, 1475.

113. Nordhoff, E., Trends Anal. Chem., 1996, 15, 240.

114. Valentine, S.J., Clemmer, D.E., J. Am. Chem. Soc., 1997, 119, 3558.

115. Konerman, L., Rosell, F.I., Mauk, A.G., Douglas, D.J., Biochemistry, 1997, 36, 6448.

116. Jarrold, M.F., Ann. Rev. Phys. Chem., 2000, 51, 179.

117. Douglas, D.J., J. Am. Soc. Mass. Spectrom., 1998, 9, 101.

118. Dong, F., Allawi, H.T., Anderson, T., Neri, B.P., Lyamichev, V.I., Nucl. Acids Res., $2001,29,3248$ 